\title{
Bypass transition in boundary layers subject to strong pressure gradient and curvature effects
}

\author{
Yaomin Zhao $^{1} \dagger$, Richard D. Sandberg ${ }^{1}$ \\ ${ }^{1}$ Department of Mechanical Engineering, University of Melbourne, VIC 3010, Australia
}

(Received $\mathrm{xx}$; revised $\mathrm{xx}$; accepted $\mathrm{xx}$ )

This paper aims at characterizing the bypass transition in boundary layers subject to strong pressure gradient and curvature effects. A series of highly resolved large-eddy simulations of a high-pressure turbine vane are performed, and the primary focus is on the effects of freestream turbulence (FST) states on transition mechanisms. The turbulent fluctuations that have convected from the inlet first interact with the blunt blade leading edge, forming vortical structures wrapping around the blade. For cases with relatively low-level FST, streamwise streaks are observed in the suction-side boundary layer, and the instabilities of the streaks cause the breakdown to turbulence. Moreover, the varicose mode of streak instability is predominant in the adverse-pressure gradient region, while the sinuous mode is more common in the (weak) favorable pressure gradient region. On the other hand, for cases with higher levels of FST, the leading-edge structures are more irregularly distributed and no obvious streak instability is observed. Accordingly, the transition onset occurs much earlier, through the breakdown caused by interactions between vortical structures. Comparing between different cases, it is the competing effect between the FST intensity and the stabilizing pressure gradient that decides the path to transition and also the transition onset, whereas the integral length scale of FST affects the scales of the streamwise streaks in the boundary layer. Furthermore, while the streaks in the low-level FST cases are mainly induced by leading edge vortical structures, the corresponding fluctuations show a stage of algebraic growth despite of the weak favorable pressure gradient and curvature.

Key words: Transition to turbulence, Compressible boundary layers, Boundary layer structure

\section{Introduction}

Laminar-turbulent transition is one of the most challenging problems in turbulence research. In particular, bypass transition (Klebanoff et al. 1962; Morkovin 1969), which bypasses the orderly process instigated by the instability of Tollmien-Schlichting waves (Herbert 1984, 1988), is usually induced by moderate or high levels of freestream turbulence (FST). Because of its wide existence in engineering applications, bypass transition has been extensively investigated, and many results from theoretical, experimental, and numerical studies have been reported (see Durbin \& Wu 2007; Schlatter et al. 2008; Zaki 2013).

$\dagger$ Email address for correspondence: yaomin.zhao@unimelb.edu.au 
It is known that bypass transition in many cases is dominated by streaky structures in the boundary layer showing alternative high- and low-speed velocity fluctuations in the streamwise direction (Westin et al. 1994; Matsubara \& Alfredsson 2001). These streaks are affected by FST, for which only low-frequency fluctuations can penetrate into the boundary layer while high-frequency modes are prohibited. This mechanism was named the shear-sheltering effect by Jacobs \& Durbin (1998). Based on the theory of optimal disturbances (Andersson et al. 1999), it was observed that streamwise vortices can cause the formation of streamwise streaks, with the maximum spatial energy growth scaling linearly with the distance from the leading edge. The streaks keep intensifying while convecting downstream which then causes the final breakdown into turbulent spots, and the physical mechanism for the amplification can be illustrated by the lift-up effects (Brandt 2014). Different breakdown paths of the streaks, including the sinuous (antisymmetric) (Andersson et al. 2001) and the varicose (symmetric) (Skote et al. 2002) modes, have been carefully investigated in the numerical simulations in Brandt et al. (2004). On the other hand, the numerical simulations in Jacobs \& Durbin (2001) showed that the breakdown of the near-wall streaks is due to interactions with the freestream disturbances when lifted up to the edge of the boundary layer, rather than being caused by the secondary instability of the streaks themselves. Based on experimental results, Mandal et al. (2010) found that the inclined shear with highly inflectional velocity profiles caused by the streaks seems to be the precursor of the turbulent spots. More recently, the concept of edge state, which originates from the dynamics of systems, has been applied in combination with the receptivity of boundary layers to explain the nucleation mechanism for turbulent spots (Khapko et al. 2016; Kreilos et al. 2016). Based on conditional statistics of the bypass transition in flat-plate boundary layers, Marxen \& Zaki (2019) found that turbulent spots can develop a core region with similar statistics to fully turbulent boundary layers, while the edge shows elevated levels of Reynolds stresses.

Despite of the growing consensus achieved on the transition mechanisms, some controversial results have been drawn from previous studies on the effects of FST on bypass transition. Brandt et al. (2004) reported direct numerical simulations of bypass transition under turbulence with different length scales. They found that with the same level of turbulence intensity, cases with larger length scales tend to have earlier transition onsets. Nevertheless, it was also presented that the spacing of the streaks is only weakly affected by the integral length scale of the freestream disturbances, and the transition mechanisms do not obviously deviate with the varying turbulence. On the other hand, Ovchinnikov et al. (2008) conducted high-quality numerical simulations and reported that the transition onsets in cases with larger length scales were delayed due to reduced receptivity. Furthermore, the transition path for the large-scale disturbance cases is through a different mechanism, other than the streak instability. Specifically, the spotlike structures are formed via the evolution of hairpin and quasi-streamwise vortices, showing the importance of the FST length scale in determining the underlying physical mechanism. Though possible reasons for the differences listed here have been discussed in Ovchinnikov et al. (2008), it certainly suggests that bypass transition is sensitive to various factors including the shape of the leading edge and the states of FST.

It is noted that the existing knowledge on transition mechanisms is mainly from studies on relatively canonical cases like flat-plate boundary layers, while the transition mechanisms can become even more intriguing when considering various factors present in more complex applications. The effects of pressure gradients on boundary layer transition were investigated experimentally in Abu-Ghannam \& Shaw (1980), showing that the transition onsets are sensitive to adverse pressure gradients (APG), especially when the FST intensity is lower than 3\%. Moreover, Gostelow et al. (1992) found that 
the transition onset is not only affected by the local pressure gradient, but also by the history of the pressure gradients in the boundary layer. Recently, Brinkerhoff \& Yaras (2015) performed numerical simulations of a flat-plate boundary layer subject to favourable and adverse pressure gradients, showing that the flow acceleration due to the favourable pressure gradient has stabilizing effects on the streaks and prevents the development of the secondary streak instability, while the adverse pressure gradient can trigger rapid transition to turbulence. Furthermore, strong adverse pressure gradients can also cause flow separations in boundary layers, and the separation-induced transition has been studied in Alam \& Sandham (2000) and Spalart \& Strelets (2000), showing different transition paths, either via linear instability or three-dimensional unsteadiness, respectively.

The effect of blunt leading-edges can also significantly affect the mechanisms of bypass transition. Goldstein \& Wundrow (1998) studied the formation of the streamwise vorticities wrapped around the leading edge, showing that the vorticies induce wakelike disturbances which are closely related to the formation of spots and break down to turbulence (Wundrow \& Goldstein 2001). More recently, the leading-edge effects were further studied in Nagarajan et al. (2007), and a path different from the streak instability was also found responsible for the breakdown. The wave packets of disturbances, which were believed to be prominent for transition mechanisms under strong FST and large leading-edge bluntness, were observed to originate from the energetic vortical structures at the leading edge.

Considering the sensitivity of the transition mechanisms to the various factors, the transition phenomena in engineering applications, which usually couple several of the factors listed above, can be much more complicated. For example, in a numerical simulation of the T106 low-pressure turbine passage, Wu \& Durbin (2001) showed the effects of the distorted wakes coming from the inlet on the suction-side transitional boundary layer. In the same configuration at a lower Reynolds number, Michelassi et al. (2002) observed Kelvin-Helmholtz instabilities responsible for the transition in the rear part of the suction-side blade. As reported for the numerical studies of a NACA-0012 airfoil (Jones et al. 2008) and a compressor blade (Zaki et al. 2010), the APG can also cause flow separations in the boundary layer, which in turn results in rapid breakdown to turbulence. Nevertheless, most of the previous studies only focused on individual factors like pressure gradient, and the effects of FST states coupling with these factors were rarely discussed. Therefore, studies on the transition mechanisms in a configuration that combines many of these critical factors are of great interest. One configuration that brings most of the above effects together in a single set-up is the high-pressure turbine (HPT).

The HPT, which is immediately downstream of the combustion chamber in gas turbines, typically experiences high levels of incoming unsteadiness and turbulence from the combustor, and the highest temperatures, pressures, and velocities anywhere in the engine. Consideration of this component at engine-relevant conditions is therefore extremely challenging for numerical simulations. Therefore, only recently have numerical simulations of HPT passages been performed (Bhaskaran \& Lele 2010; Wheeler et al. 2016) to reveal the complex flow physics. However, the previous simulations had very limited spanwise extent, thus the length scales of the inlet turbulence were too small to represent realistic conditions.

In the present study, we investigate the bypass transition in an HPT working at conditions representative of a modern aircraft engine (Sandberg \& Michelassi 2019). By performing highly resolved simulations with up to $8.6 \times 10^{8}$ grid points, the flow physics can be investigated in detail. In particular, the effects of the FST with strong intensities and large integral length scales, which resemble the flow conditions in realistic 


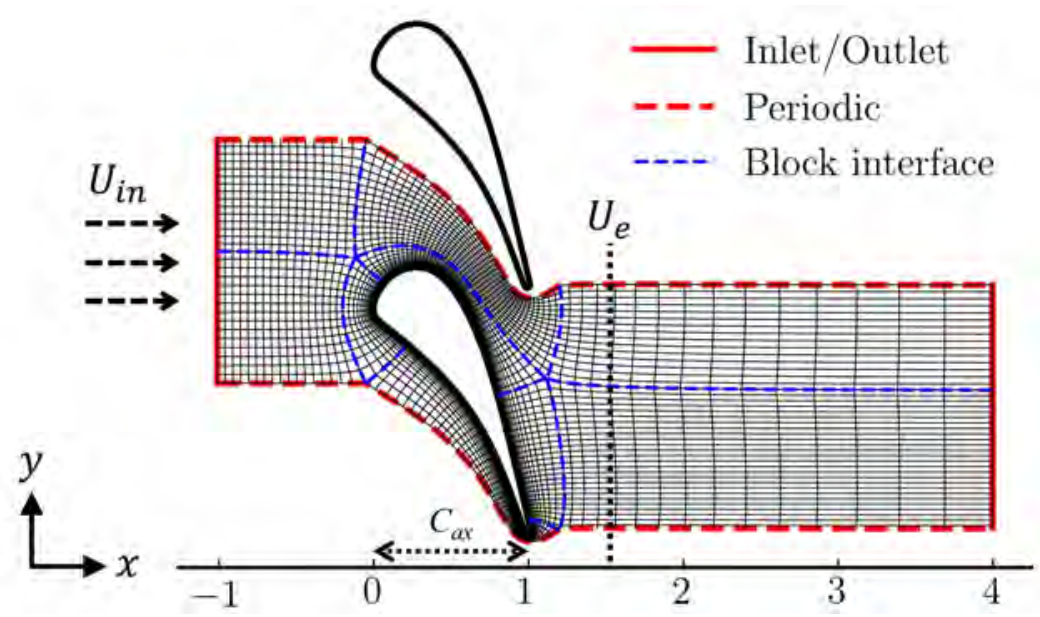

FiguRE 1. Schematic for HPT case setup. The computational grid is showing every fifteenth lines in $x$ and $y$ directions.

applications (Nix 2004), will be discussed. In the present simulations, the FST and the boundary layer are significantly affected by the strong pressure gradient and highcurvature around the blade, which makes the boundary layer transition more challenging to understand. Furthermore, by varying the intensities and integral length scales of the incoming turbulence in different cases, we are able to investigate the effects of FST states on the transition mechanisms in the blade boundary layer.

The outline of this paper is as follows. An introduction to the numerical simulations is given in $\S 2$, with a description of the inlet turbulence and validation of the results also presented. Then an overview of the flow fields obtained from the HPT simulations is given in $\S 3$, and the blade boundary layer is divided into different characteristic regions. In $\S 4$, we focus on the transition mechanisms in the suction-side boundary layer, and discuss the different paths to turbulence observed in the present cases. In addition, a discussion on the effects of the incoming turbulence on the transitional behaviours is provided in $\S 5$, and the results are compared to previous studies in flat-plate boundary layer with zero-pressure gradient. Conclusions are drawn in $\S 6$.

\section{Numerical Simulations}

\subsection{Case setup}

A schematic for the setup of the HPT simulations is shown in figure 1. A linear cascade setup is used to replicate the experiments performed at the Von-Karman Institute (VKI). In the present simulations, only one passage is calculated, and the computational domain is enclosed by the red lines on the axial and pitchwise $(x-y)$ plane cut. Representative of a modern transonic HPT nozzle, the simulations are performed at a Reynolds number of $R e=U_{e}^{*} C_{a x}^{*} / \nu^{*}=5.7 \times 10^{5}$ and an exit Mach number $M a_{e}=0.92$, which are in close agreement with the MUR224 case in the VKI experiments (Arts et al. 1990). Here, the superscript $*$ denotes dimensional quantities. Accordingly, $C_{a x}^{*}$ is the axial chord length, and $\nu^{*}$ and $U_{e}^{*}$ are the kinematic viscosity and the velocity at the exit plane, respectively.

The non-dimensionalized three-dimensional compressible Navier-Stokes equations are 
solved using the in-house solver HiPSTAR

$$
\left.\begin{array}{l}
\frac{\partial \rho}{\partial t}+\frac{\partial\left(\rho u_{j}\right)}{\partial x_{j}}=0, \\
\frac{\partial\left(\rho u_{i}\right)}{\partial t}+\frac{\partial\left(\rho u_{i} u_{j}+p \delta_{i j}\right)}{\partial x_{j}}=\frac{\partial \tau_{i j}}{\partial x_{j}}, \\
\frac{\partial\left(\rho e_{0}\right)}{\partial t}+\frac{\partial\left[u_{j}\left(\rho e_{0}+p\right)\right]}{\partial x_{j}}=\frac{\partial\left(\tau_{i j} u_{i}\right)}{\partial x_{j}}-\frac{\partial q_{j}}{\partial x_{j}} .
\end{array}\right\}
$$

Here, $\rho, u_{i}, p$ and $T$ are the non-dimensionalized flow density, velocity components, pressure and temperature, respectively. The non-dimensionalization results in dimensionless parameters as $R e_{\infty}=\frac{\rho_{\infty} U_{\infty} L_{\infty}}{\mu_{\infty}}$ and $M a_{\infty}=\frac{U_{\infty}}{c_{\infty}}$. The reference length scale $L_{\infty}$ is selected as $C_{a x}^{*}$, and the reference velocity $U_{\infty}$ and density $\rho_{\infty}$ are selected as the mean velocity and density at the inlet. Therefore, as non-dimensional quantities we have $C_{a x}=1, U_{i n}=1$, and $\rho_{i n}=1$, and the non-dimensionalized flow-over time is $t_{F}=C_{a x} / U_{i n}=1$. Moreover, $\mu_{\infty}$ and $c_{\infty}$ are viscosity and acoustic velocity for the reference state which are only dependent on the reference temperature $T_{\infty}$. The total energy $e_{0}$ is given by

$$
e_{0}=\frac{1}{2} \rho u_{i} u_{i}+\frac{T}{\gamma(\gamma-1) M a_{\infty}^{2}},
$$

where $\gamma=1.4$ is the specific heat ratio. Moreover, the stress tensor is written as

$$
\tau_{i j}=\sigma_{i j}+\tau_{i j}^{s g s}
$$

which includes the viscous stress $\sigma_{i j}=\frac{\mu}{R e_{\infty}}\left(\frac{\partial u_{i}}{\partial x_{j}}+\frac{\partial u_{j}}{\partial x_{i}}-\frac{2}{3} \frac{\partial u_{k}}{\partial x_{k}} \delta_{i j}\right)$ and the sub-grid scale (SGS) stress $\tau_{i j}^{s g s}$. The molecular viscosity $\mu$ is computed using Sutherlands law (White 1991), setting the ratio of the Sutherland constant over freestream temperature to 0.36867 . Similarly, the heat flux $q_{j}$ is written as

$$
q_{j}=-\frac{\mu}{(\gamma-1) \operatorname{Pr} R e_{\infty} M a_{\infty}^{2}} \frac{\partial T}{\partial x_{j}}+q_{j}^{s g s},
$$

with $\operatorname{Pr}=0.72$ representing the Prandtl number. The SGS terms in the governing equations, i.e. SGS stress $\tau_{i j}^{s g s}$ and heat flux $q_{j}^{s g s}$, are closed by the WALE model by Nicoud \& Ducros (1999). A fourth-order wavenumber-optimized compact finite difference scheme (Kim \& Sandberg 2012) was applied in the $x$ and $y$ directions, while a spectral method using fast Fourier transforms was applied in the uniform spanwise direction $z$. Moreover, the ultra-low storage frequency optimized explicit Runge-Kutta method (Kennedy et al. 2000) was used for time integration. Therefore, the numerical schemes used in the present simulations ensure fourth or higher-order accuracy of both spatial and temporal discretizations.

In order to efficiently adapt the complex geometry without affecting the numerical accuracy, the computational grid of the present configuration is decomposed into nine blocks, and the characteristic interface conditions (Kim \& Lee 2003) were employed at the block interfaces as presented by the blue dashed lines in figure 1 . This multiblock setup consists of an O-type grid around the blade and a background H-type grid, which allows accurately resolving the blade boundary layers and enforcing the pitchwise periodicity at the same time. Moreover, a characteristic boundary condition with a sponge layer is applied at the outlet to prevent spurious reflection (Sandberg \& Sandham 2006), and the blade surface is set as non-slip iso-thermal wall. At the inlet, a Riemann boundary condition is applied, and fluctuations, as described in $\S 2.2$, are 


\begin{tabular}{lccccccc} 
Cases & $T_{u} / U_{\text {in }}$ & $L_{s} / C_{a x}$ & $L_{z}$ & $\left\langle\Delta s^{+}\right\rangle$ & $\left\langle\Delta n_{w}^{+}\right\rangle$ & $\left\langle\Delta z^{+}\right\rangle$ & Total points \\
\hline A & $6.5 \%$ & $5 \%$ & 0.3 & 25.6 & 1.8 & 10.0 & $3.2 \times 10^{8}$ \\
B & $6.5 \%$ & $20 \%$ & 0.8 & 25.1 & 1.8 & 9.8 & $8.6 \times 10^{8}$ \\
C & $20 \%$ & $5 \%$ & 0.3 & 27.1 & 1.9 & 10.5 & $3.2 \times 10^{8}$ \\
D & $20 \%$ & $20 \%$ & 0.8 & 30.6 & 2.1 & 11.5 & $8.6 \times 10^{8}$
\end{tabular}

TABle 1. Parameters for HPT cases.
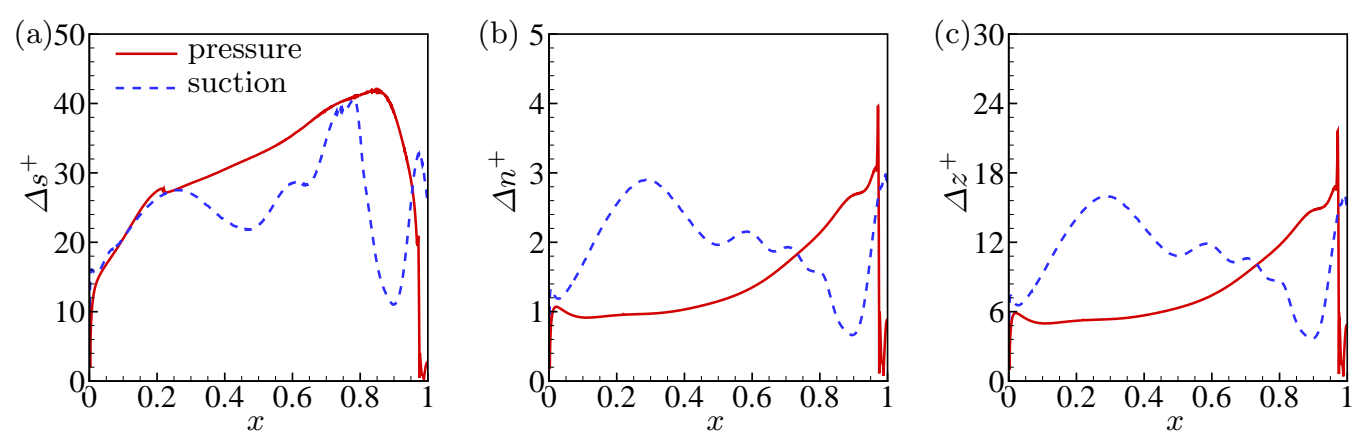

Figure 2. Near-wall grid size along the blade surface measured in wall units for case A, (a) in the tangential direction $\Delta s^{+}$; (b) in the wall-normal direction $\Delta n^{+}$; (c) and in the spanwise direction $\Delta z^{+} ;$.

added to simulate the turbulence coming from upstream combustors in real engines. It is noted that the numerical solver and the multi-block setup for turbine cascades have been extensively validated in previous studies, and more details can be found in Sandberg et al. (2015).

In order to investigate the effects of the incoming turbulence on the HPT performance, four LES cases with different turbulence intensities and length scales have been performed in the present study, and the parameters of the cases are summarized in table 1 . It is noted that the integral turbulence length scale $L_{s}$ introduced in cases B and D is as large as $20 \% C_{a x}$, which requires a much larger domain in the spanwise direction compared to previous studies (Bhaskaran \& Lele 2010; Wheeler et al. 2016). Therefore, the number of grid points significantly increases, and the final meshes applied in the present simulations have up to $8.6 \times 10^{8}$ grid points. Furthermore, the grid resolution around the blade boundary layer for case A is shown in figure 2. The grid spacings on the blade surface in the tangential, wall-normal, and spanwise directions are normalized with the local viscous length scale $\delta_{\nu}=\nu / u_{\tau}$ and denoted as $\Delta s^{+}, \Delta n^{+}$, and $\Delta z^{+}$, respectively. Here $u_{\tau}=\sqrt{\tau_{w} / \rho}$ is the wall friction velocity with the wall shear stress $\tau_{w}$ and density $\rho$. Although the grid sizes vary along the blade surface, they remain at a relatively low level, and the grid spacings averaged around the blade surface are also given in table 1. It is also noted that the spanwise extents of the present cases, with the smaller ones being $L_{z}=0.3$ for cases A and C, are around 6 times of the maximum boundary layer thickness around the blade. By comparing case A to the DNS results of the same configuration with a much narrower spanwise extent $\left(L_{z}=0.1\right)$ (Wheeler et al. 2016), the wall heat flux and other boundary layer statistics are in close agreement, showing that the current grid resolution is sufficient to accurately resolve the boundary layer and most of the energy-containing scales for the FST (Pichler et al. 2017). 
(a)

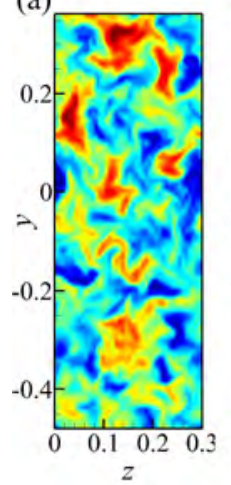

(b)

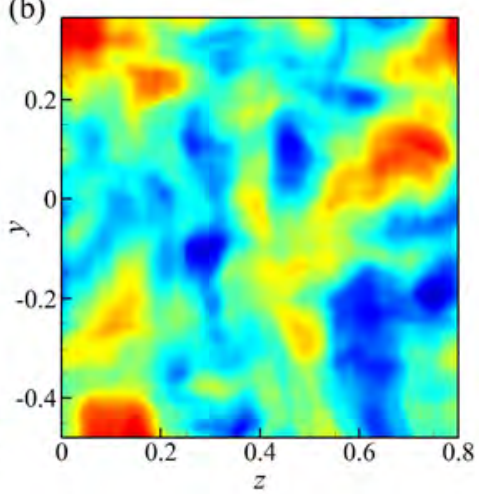

(c)

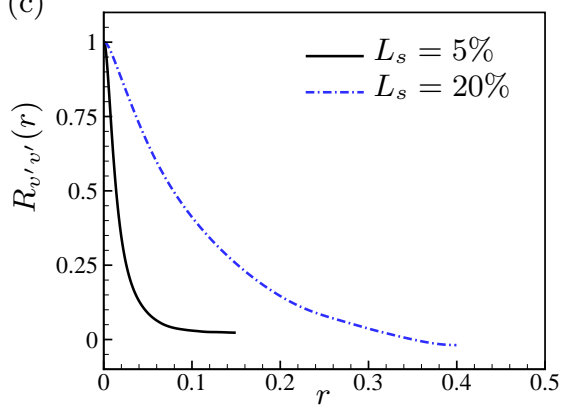

FiguRE 3. Incoming turbulence at a plane cut upstream of the blade leading edge at $x=-0.5$. The contours of the instantaneous streamwise velocity component from cases A and B are presented in figures (a) and (b), respectively. The spanwise correlation of the pitchwise fluctuating velocity $v^{\prime}$ is shown in figure (c).

\subsection{Inlet turbulence}

The turbulent fluctuations at the inlet are introduced by a digital filter method (Klein et al. 2003), in which the generated velocity fields can efficiently reproduce first and second order one-point statistics as well as a locally given autocorrelation function. Therefore, the turbulence intensities and integral length scales can be prescribed for the different cases given in table 1 . In order to investigate the generated inlet turbulence formed in cases with different length scales, the contours of instantaneous streamwise velocity at a $y-z$ plane cut at $x=-0.5$ are presented in figure 3 . It is shown that the turbulent structures formed in case B, with a prescribed $L_{s}=20 \% C_{a x}$, indeed have much larger scales compared to the structures in case A, with a prescribed $L_{s}=5 \% C_{a x}$. Furthermore, the spanwise correlation of the pitchwise fluctuating velocity $R_{v^{\prime} v^{\prime}}(r)$ is also computed at this plane-cut for cases A and B and shown in figure 3(c). The integral turbulence length scales, therefore, can be estimated as $2 \int_{0}^{L_{z} / 2} R_{v^{\prime} v^{\prime}}(r) d r$, and the results for cases $\mathrm{A}$ and $\mathrm{B}$ are $4.8 \% C_{a x}$ and $20.1 \% C_{a x}$, respectively. Thus, it is shown that the integral length scales of the incoming turbulence are in close agreement with the prescribed values. In addition, it can also be inferred that the spanwise extents for the presented simulations are sufficient for the large-scale inlet turbulence as

$$
\lim _{r \rightarrow L_{z} / 2} R_{v v}(r) \rightarrow 0
$$

The length scale of the prescribed inlet turbulence not only affects the large-scale structures formed at the inlet, but also influences the decay rate of the incoming turbulence. This can be shown by the evolution of the pitchwise averaged turbulence intensity

$$
T_{u}=\sqrt{\frac{u^{\prime 2}+v^{\prime 2}+w^{\prime 2}}{3}}
$$

in figure 4. The turbulence intensities at the inlet are set as $6.5 \% U_{\text {in }}$ and $20 \% U_{\text {in }}$ respectively. It can be observed that the cases with larger integral length scale $\left(L_{s}=\right.$ $\left.20 \% C_{a x}\right)$ have a lower dissipation rate compared to the ones with smaller scale $\left(L_{s}=\right.$ $\left.5 \% C_{a x}\right)$. Before entering into the vane, the intensities at $x=-0.2$ for the different cases reach levels of $16.8 \%, 9.5 \%, 6.1 \%$ and $5.2 \%$ of the inlet velocity $U_{i n}$, respectively. 

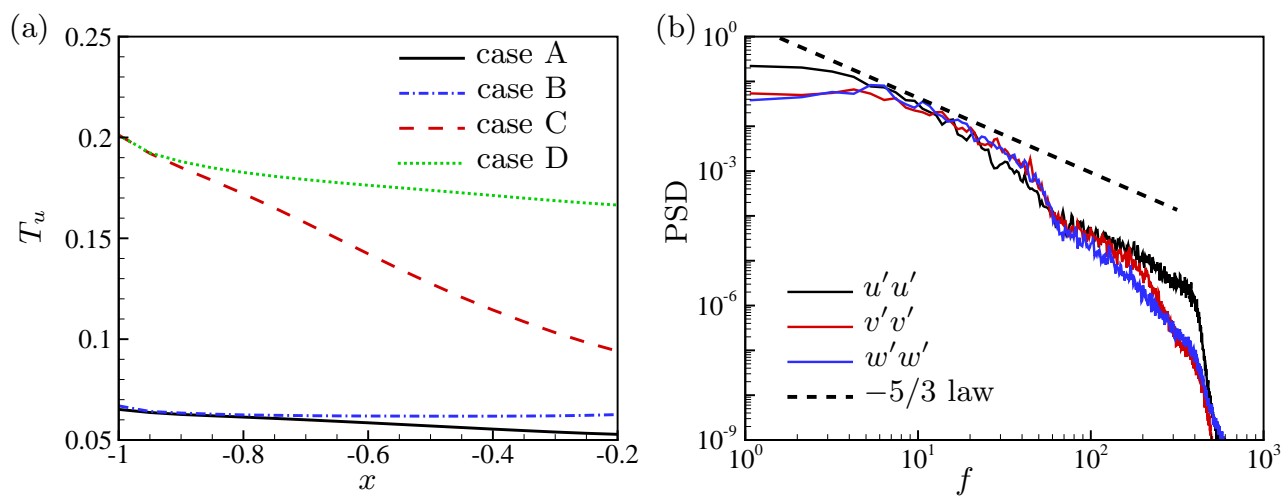

FIgURE 4. Characterization of the inlet turbulence: (a) evolution of pitchwise averaged turbulence intensity $T_{u}$ along the streamwise direction; (b) PSD of different velocity components normalized by $T_{u}^{2}$ in case A.

Furthermore, the turbulence spectra at a monitor point upstream of the blade leading edge $(x=-0.2, y=0.0)$ have been extracted, and the power spectral density (PSD) of the three fluctuating velocity components for case A are presented in figure 4(b). The curves for all of the three components show a range of $-5 / 3$ behaviour, indicating an inertial range for the incoming turbulence. Moreover, it is noted that the streamwise component is stronger at low frequencies compared to the other components, which implies that the turbulence turns anisotropic downstream of the inlet due to the streamwise flow acceleration, driven by the pressure ratio between the outlet and inlet.

\subsection{Validation with experiments}

The results from the present simulations, particularly the accuracy of the prediction of the blade boundary layers, have been quantitatively validated against the available data from the VKI experiments (Arts et al. 1990). The isentropic Mach number $M a_{i s}$, which is related to the pressure distribution around the blade boundary layer, is plotted against the surface length $s$ in figure 5(a). On the pressure-side of the blade $(s<0), M a_{i s}$ increases monotonously from the leading edge at $s=0$, which indicates a favourablepressure gradient (FPG) boundary layer across the pressure-side blade, as shown in figure $7(\mathrm{a})$. On the other hand, $M a_{i s}$ on the suction-side surface $(s>0)$ first increases and then decreases, and the peak of $M a_{i s}$ divides the suction-side boundary layer into the FPG and APG regions. While the $M a_{i s}$ distribution for the same $R e$ was not reported in the experiment, an experimental case with the same pressure ratio but at a higher-Re is also selected for comparison in figure 5(a). It is shown that the numerical results are in close agreement with the experimental data, and the cases with different inlet turbulence do not show significant deviation for the mean pressure distribution around the blade.

Furthermore, the blade surface heat flux

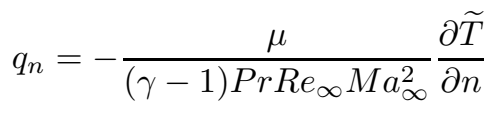

is also presented in figure $5(\mathrm{~b})$. Here, $\widetilde{T}$ is the Favre-averaged temperature. The experimental data sets shown here are from two cases at the same Re and $M a$, while the turbulence intensities are at the levels of $4 \% U_{i n}$ and $6 \% U_{i n}$, respectively. As the turbulence length scales or dissipation rate were not provided in the experiments, it is not possible to reproduce the exact turbulence states in the numerical simulations (Bhaskaran 

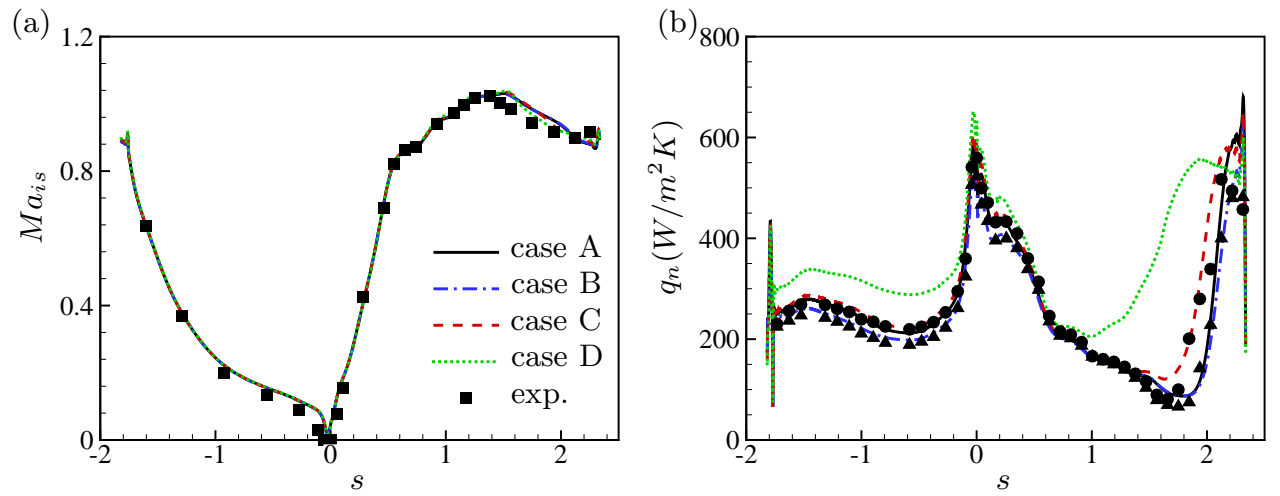

FiguRE 5. Validation of the results: (a) isentropic Mach number $M a_{i s}$ across the blade surface, for negative $s$ on the pressure-side and for positive $s$ on the suction-side; (b) blade surface heat flux $q_{n}$, symbols representing experimental data sets with $T_{u}$ levels at $6 \%$ and $4 \%$.

\& Lele 2010). However, despite the potential differences of the boundary conditions, the heat flux distributions from cases $\mathrm{A}, \mathrm{B}$ and $\mathrm{C}$ agree well with the experimental data, including the prediction of the transition onset. Moreover, the case D with highintensity and large-scale inlet turbulence, which is more representative of the real engine environments, shows significant deviation from other cases, and it is therefore of great interest in the present study.

\section{Overview of the Flow Field}

An overview of the HPT flow field is given to present the complex flow phenomena through the passage. Contours of the mean velocity magnitude $U$, along with the isolines of the mean pressure $\bar{p}$, are shown in figure $6(\mathrm{a})$. Through the HPT vane, the flow accelerates significantly due to the pressure ratio between the inlet and the outlet. From the blade leading edge, it first passes through a region of high-curvature and strong pressure gradient marked by the arrow $M 1$. Then the flow acceleration continues until the minimum of $\bar{p}$, marked by the arrow $M 2$, which is followed by the APG region on the blade suction side. Moreover, a pressure dipole marked by $M 3$ is caused by the vortex shedding at the blade trailing edge.

Furthermore, a snapshot of the density gradient $|\nabla \rho|$ is presented in figure $6(\mathrm{~b})$ to show the instantaneous flow structures in case B. Large-scale structures, formed due to the inlet turbulence, interact with the blade leading edge and then enter into the vane. The structures are stretched because of the strong FPG, especially near the region $M 1$ shown in figure 6(a), and both the suction-side and pressure-side boundary layers are affected. Moreover, scattered shocks are observed on the blade suction-side, which are located near the minimum of $\bar{p}$, denoted by $M 2$ in figure 6(a). Further downstream, the vortex shedding at the blade trailing-edge induces a wake region with strong mixing, and the acoustic waves caused by the shedding and its interaction with the blade surface propagate upstream and to the adjacent blade and thus affect the suction-side boundary layer.

The blade boundary layer is further characterized in figure 7 based on the surface curvature $\kappa$, the pressure gradient along the surface normalized by the wall-friction velocity $u_{\tau}$

$$
\Delta P=\nu \frac{\partial \bar{p}}{\partial s} / u_{\tau}^{3}
$$



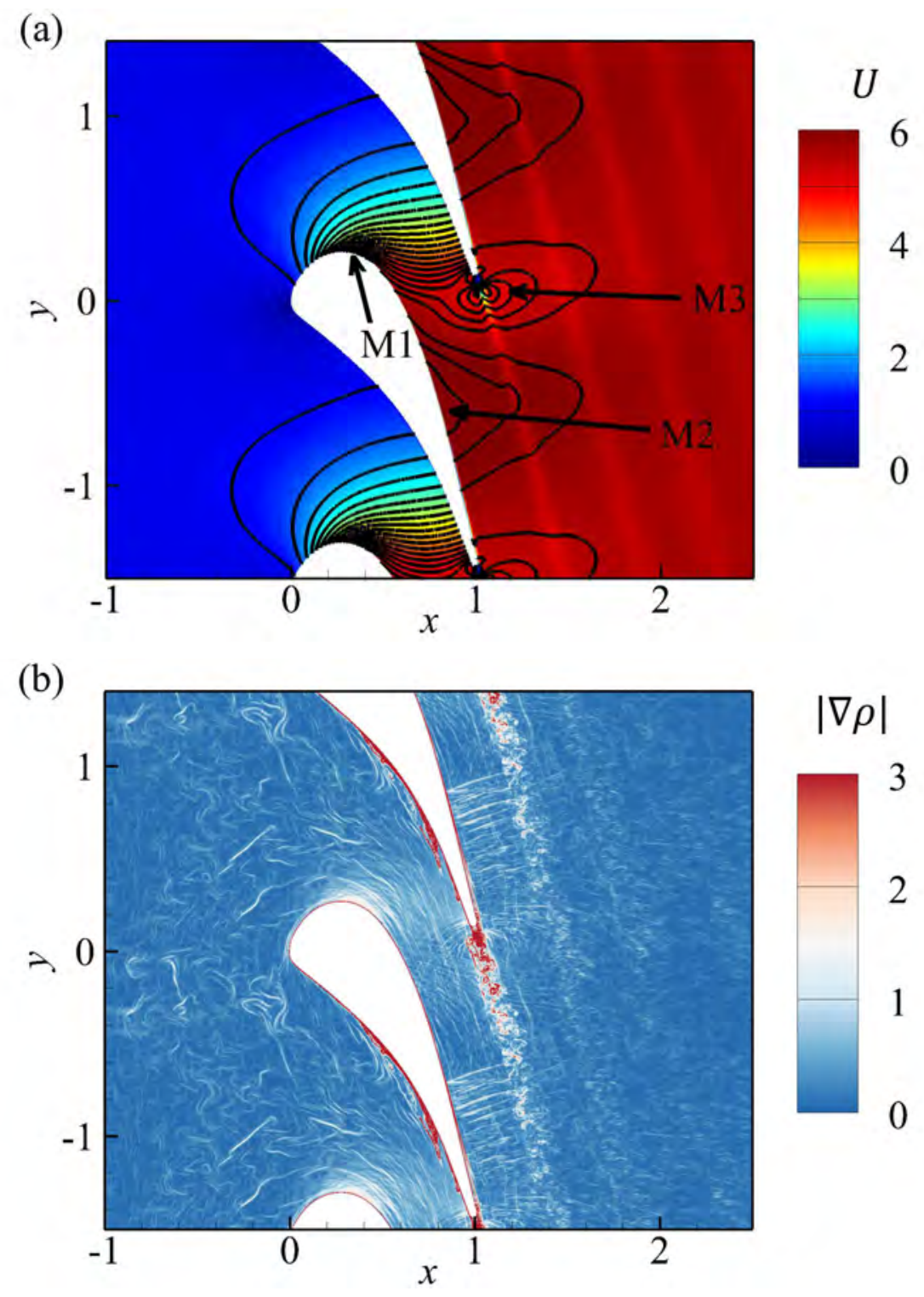

Figure 6. Overview of the flow field: (a) contour of the mean velocity magnitude $U$ with iso-lines of time-averaged pressure $\bar{p}$; (b) contour of the instantaneous density gradient $|\nabla \rho|$.

and the wall friction coefficient

$$
C_{f}=\frac{2 \tau_{w}}{\rho_{i n} U_{i n}^{2}} .
$$

The blade surface is first divided into the suction-side and pressure-side by the leading edge (LE) and trailing edge (TE) as shown in figure 7(a). For the pressure-side boundary layer, the amplitude of curvature is relatively low, and the pressure gradient has a nearconstant value of $\Delta P \approx-0.025$, except for a short region near the LE, which is considered to have strong stablizing effects (Patel 1965; Araya et al. 2015). Accordingly, the $C_{f}$ for the pressure-side increases gradually and implies a laminar state boundary layer, even 
(a)
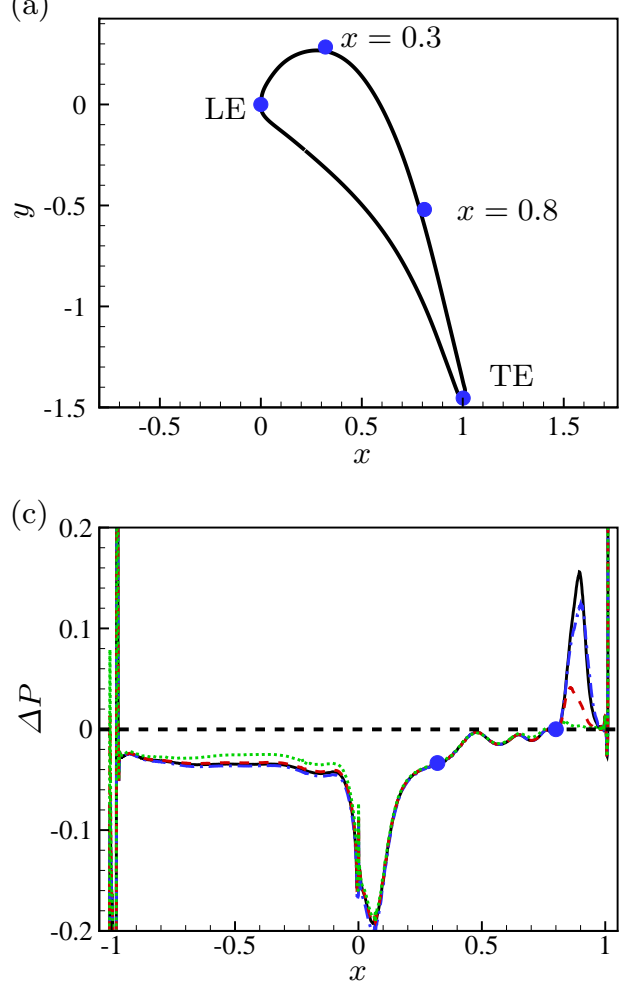

(b)

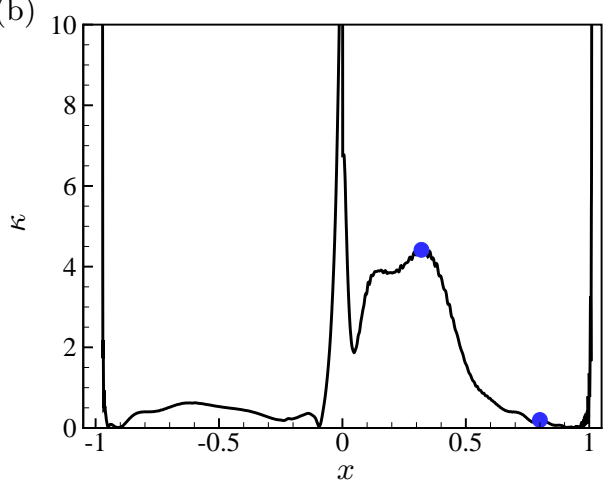

(d)

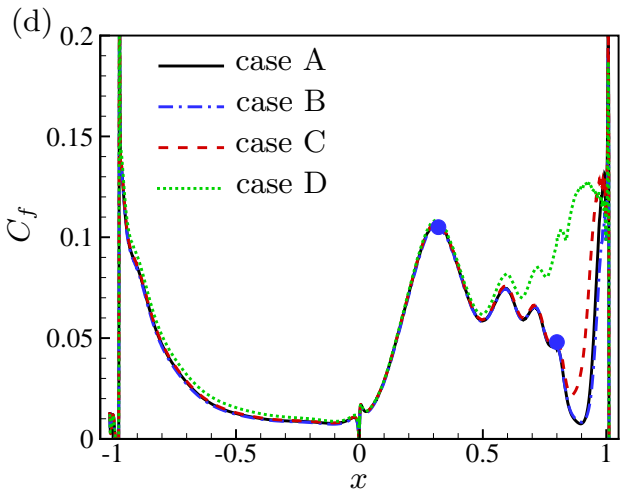

Figure 7. Mean flow statistics for the HPT simulations: (a) blade boundary layer divided by critical points marked by the blue circles; (b) blade surface curvature $\kappa$; (c) pressure gradient normalized by wall-friction velocity $\Delta P$; (d) wall-friction coefficient $C_{f}$.

for case D with the largest amplitude FST. Therefore, the pressure side boundary layers are not further analysed in the current study.

On the other hand, the suction-side boundary layer is characterised by varying behaviours in different regions. We therefore divide the boundary layer into regions dominated by different factors, and the boundaries of the regions are indicated by the blue filled circles in figure 7 . For the first stage, from the stagnation point at $x=0.0$ to the suction peak at $x=0.3$, the blunt leading edge region is dominated by the high convex curvature and strong FPG, and the mean flow of the boundary layer at this stage stays laminar despite of the disturbances caused by the turbulence coming from the inlet for all of the cases, as shown by the wall-friction in figure $7(\mathrm{~d})$. In the second stage of the suctionside boundary layer, from $x=0.3$ to the pressure minimum at $x=0.8$, the curvature and the FPG gradually decrease. While for cases A-C the wall friction coefficient $C_{f}$ remains laminar in this region, in case D, which is affected by the largest-amplitude FST, shows transtion to turbulence at this stage. Further downstream, the last stage, from $x=0.8$ to the TE, is the APG boundary layer with negligible curvature. The boundary layers for all of the cases transition to turbulence in this APG region, while the stronger FST in case $\mathrm{C}$ induces a slightly earlier transition. A summary of the different regions of the suction-side boundary layer is also presented in table 2 . 


\begin{tabular}{llll} 
Stages & Coordinates & \multicolumn{1}{c}{ Features } & Boundary layer state \\
I & $x=0.0 \sim 0.3$ & Strong FPG and high curvature & Laminar mean flow \\
II & $x=0.3 \sim 0.8$ & Decreasing FPG and curvature & Transition: case D \\
III & $x=0.8 \sim 1.0$ & APG and negligible curvature & Transition: cases A/B/C
\end{tabular}

TABLE 2. Different regions of the suction-side boundary layer.

\section{Boundary Layer Transition}

In this section, we focus on the suction-side boundary layer transition. An overview of the instantaneous boundary layer flows for all of the cases is first presented in figure 8, and the vortical structures are shown by iso-surfaces of the $Q$ criterion (Hunt et al. 1988). In addition, the contours of the tangential velocity fluctuation $u_{t}^{\prime}$ on a surface parallel to the blade are also presented, and the distance between the surface to the blade is around $\delta / 2$ at $x=0.5$, with $\delta$ representing the boundary layer thickness reaching $99 \%$ of the local freestream mean velocity. It is obvious that the boundary layers show diverse transitional behaviours under the different states of incoming turbulence. For cases with relatively low-level turbulence, i.e. cases A and B, streamwise vortical tubes form in the leading-edge region $(x<0.3)$ and then stretch downstream in the FPG part of the boundary layer $(0.3<x<0.8)$. Further downstream, the vortical structures quickly break down into turbulence in the APG region $(x>0.8)$. At the same time, low-speed streaks, which are considered as dominant structures in bypass transition induced by FST (Durbin \& Wu 2007), are also shown to be present in these cases. For cases C and $\mathrm{D}$ featuring increasing amplitude incoming turbulence, the vortical structures are more 'chaotic', and the instability and meandering of the low-speed streaks tend to appear farther upstream. Accordingly, the transition onsets occur earlier compared to the cases with lower-level inlet turbulence, which is also shown by the wall-friction coefficient in figure $7(\mathrm{~d})$.

In the following subsections, the mechanisms for the suction-side boundary layer transition will be discussed, with emphasis on the various phenomena caused by the different FST characteristics. We will first investigate the effects of the blunt leading edge, in which vortical structures start to wrap around the blade and streak-like structures form in the boundary layer. Thereafter, several key mechanisms responsible for the breakdown to turbulence will be presented.

\subsection{Leading-edge structures}

As shown by the blade geometry in figure 1, the HPT in the present simulations has a blunt leading edge, which is known to have direct effects on the receptivity process of the boundary layer (see Kendall 1991). In addition, it has been reported that streamwise vortices can form around the leading edge, which is due to the tilting and stretching of the incoming wall-normal vortices (Goldstein \& Wundrow 1998; Wundrow \& Goldstein 2001). The streamwise vortices thus can induce wake-like disturbances similar to backward jets (Jacobs \& Durbin 2001) or low-speed streaks (Brandt et al. 2004), and the instability of these streamwise streaks finally results in the breakdown to turbulence. Other than the instability of streaks, Nagarajan et al. (2007) introduced a different path to transition considering the effects of the leading edge, for which turbulent spots are formed via precursors which can be traced back to leading-edge structures. This breakdown mechanism due to leading-edge structures was observed to be dominant when the FST intensity or the leading-edge bluntness is large. In the present study, 
(a)

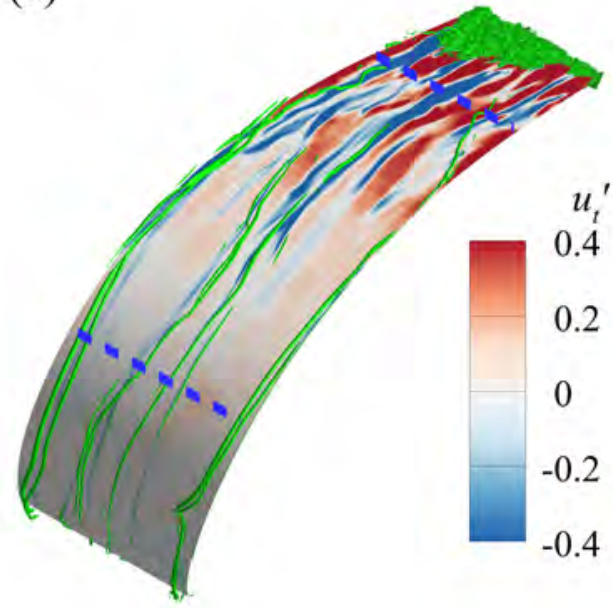

\section{(c)}

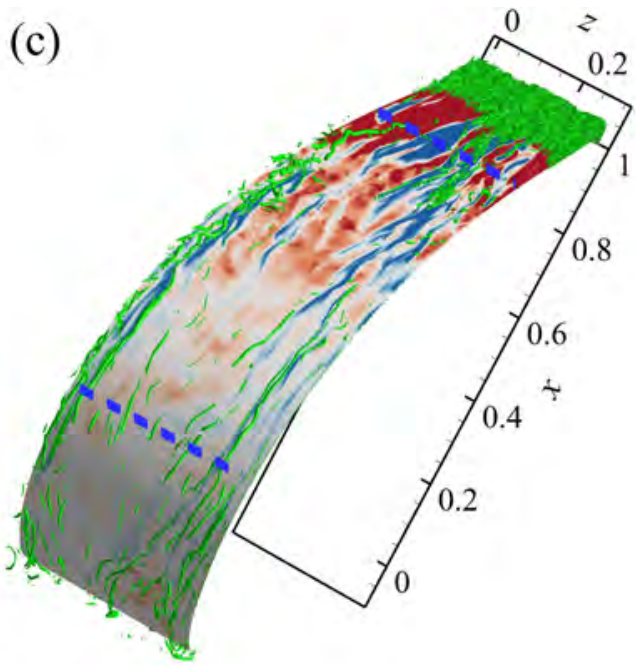

(b)

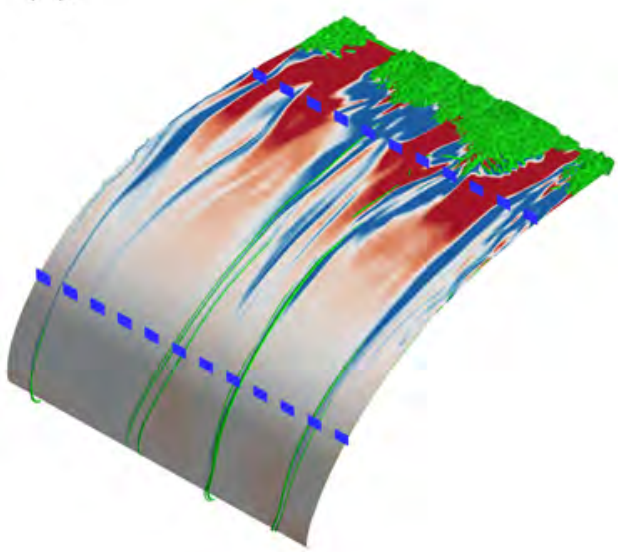

(d)

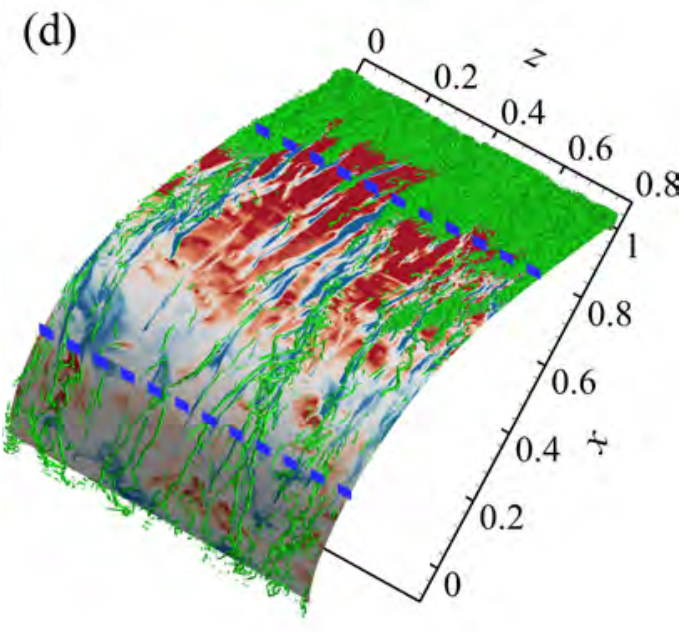

FiguRE 8. The vortical structures on the suction-side boundary layer: (a) case A; (b) case B; (c) case $\mathrm{C}$; and (d) case D. Instantaneous iso-surfaces of $Q=4000$ are presented, and contours of the tangential velocity perturbations $u_{t}^{\prime}$ on a surface parallel to the blade are also shown. The distance between this surface to the blade is around $\delta / 2$ at $x=0.5$. In addition, the blue dashed lines indicate the locations of $x=0.3$ and $x=0.8$.

the extraordinarily blunt leading edge of the HPT, along with incoming turbulence of high amplitudes and large scales coming from the inlet, is considered. It is clearly shown in figure 8 that the boundary layer transition taking place in all of the cases is influenced by the vortical structures from the leading-edge region. Therefore, the leadingedge structures will be studied to help understand the downstream transition.

The basic boundary layer statistics, i.e. the profiles of the mean tangential velocity and the velocity fluctuations at different locations, are presented in figure 9 . In particular, the cases A and D, with the lowest and highest levels of inlet turbulence in the present study, are selected for comparison. As shown in figure 9(a), the mean velocity profiles for both cases are in close agreement with each other, despite the remarkably strong fluctuations case $\mathrm{D}$ is subjected to, as presented in figure $9(\mathrm{~b})$. Furthermore, the mean tangential 
(a)

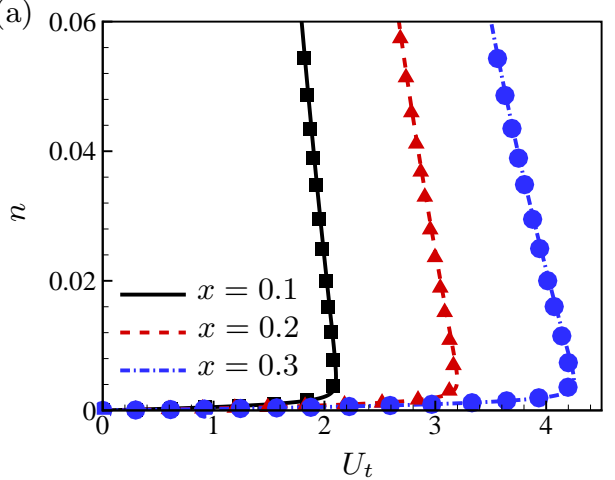

(b)

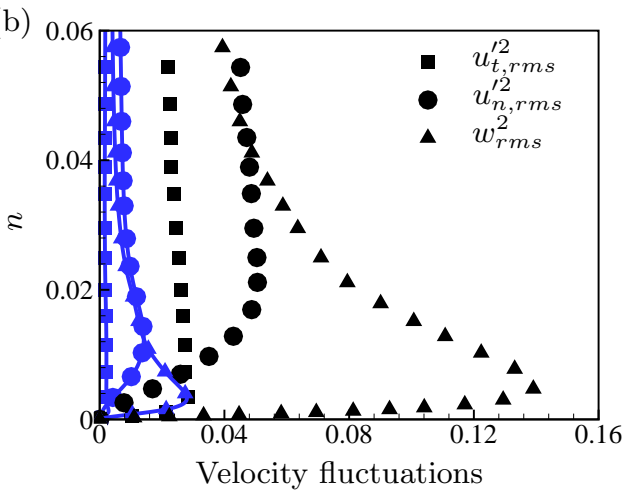

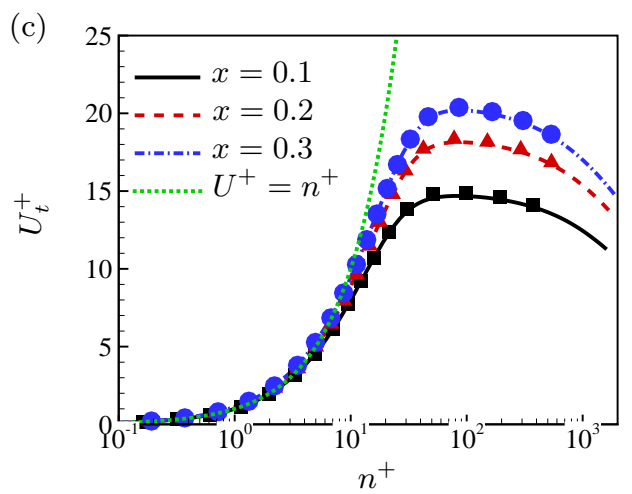

FIgURE 9. Wall-normal profiles in the leading-edge region. (a) Mean tangential velocity $U_{t}$ at different locations for case A (lines) and case D (symbols); (b) velocity fluctuations at the leading edge $x=0.0$ for case A (lines and symbols) and case D (symbols only); (c) mean tangential velocity profiles in wall-units for case $\mathrm{A}$ (lines) and two-dimensional laminar flow (symbols).

velocity profiles in case A are plotted in wall-units in figure 9(c), in comparison with the profiles from a two-dimensional case without any FST, and the close agreement implies that the boundary layers in the leading-edge region $(x<0.3)$ stay laminar. This is due to the stabilizing effects of the strong FPG and high convex curvature (see Muck et al. 1985; Katz et al. 1990; Mukund et al. 2006), which is prominent in the leading-edge region as identified in figure $7(\mathrm{~b})$ and $(\mathrm{c})$.

It is also demonstrated in figure 9 that compared to other components, the amplitude of the spanwise fluctuation is much larger near the wall in both cases. The strong spanwise disturbances at the leading edge are also shown by instantaneous contours of the spanwise velocity component shown in figure 10(a), and a schematic for this plane cut is given in figure 10(b). It appears that the large amplitude of tangential vorticity, presented by the iso-lines, is basically located at those regions showing strong gradients of the spanwise velocity fluctuation $w^{\prime}$. This relation between the large amplitude of spanwise disturbances and tangential vorticity can be expressed as $\omega_{t}^{\prime} \sim \frac{\partial w^{\prime}}{\partial n}$, with $n$ representing the wall-normal distance to the blade surface. Similar to the observation in Goldstein \& Wundrow (1998), the tangential vortices at the leading edge will wrap around the blade surface, and the resulting streamwise vortices are expected to play a key role in downstream transition.

The effects of the leading-edge structures on the boundary layer are further investigated by scrutinizing flow variables in the wall-normal-spanwise planes, as shown in figure 11 . 
(a)

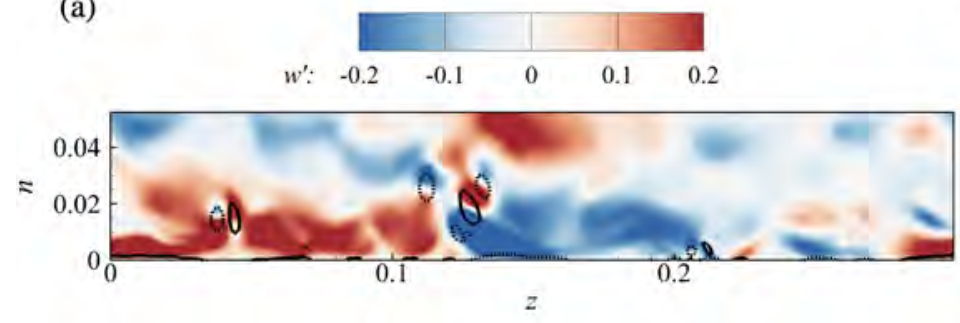

(b)

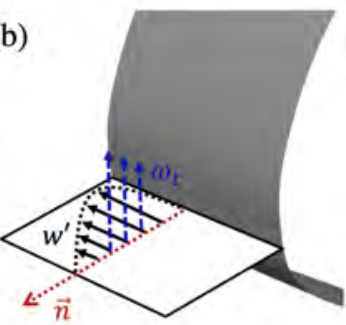

Figure 10. (a) Contour of spanwise velocity fluctuation at the lead-edge wall-normal plane cut in case A. Iso-lines of tangential vorticity $\omega_{t}^{\prime}$ (normal to the plane cut) are also shown, with solid and dashed lines indicating $\omega_{t}^{\prime}=100$ and $\omega_{t}^{\prime}=-100$, respectively. (b) Schematic for the wall-normal plane cut at the leading edge.

In the wall-normal plane cut near the suction peak at $x=0.3$, the iso-lines of $Q$ represent the streamwise vortical structures wrapped around the leading edge, which are also shown in figure 8 , while the contours show the tangential velocity fluctuation $u_{t}^{\prime}$ and the temperature fluctuation $T^{\prime}$, respectively. For case A, it is noted that the nearwall region as marked by the black dashed box in figure 11(a) is packed with strips of positive and negative velocity fluctuations. In particular, the low-speed fluid seems to be elevated and forms 'mushroom-like' structures, which are also shown by the contour of temperature fluctuation $T^{\prime}$ from figure 11(c). The lift-up effects of the vortical structures are clearly presented in figures $11(\mathrm{~d})$ and (e), in which two 'mushroom-like' structures in figure 11(c) are extracted to provide a zoom-in view, with the vectors showing the velocity fluctuations in the spanwise $w^{\prime}$ and wall-normal $u_{n}^{\prime}$ directions. It appears that the vortical structures induce strong upward velocity near the wall, driving the fluid with relatively low temperature away from the blade and thus enhancing the heat flux. Therefore, the streamwise vortices are proven to be directly linked to the low-speed streaks, which has also been presented in several previous studies on flat plate boundary layers with zero pressure gradients (ZPG) (Andersson et al. 2001).

For comparison, the contour of $u_{t}^{\prime}$ in figure 11(b) shows the velocity fluctuations in case D, which are apparently much more violent compared to those in case A. Moreover, the iso-lines of $Q$ are nearly randomly distributed over the plane cut, corresponding to the 'chaotic' vortical structures as shown in figure $8(\mathrm{~d})$. This indicates that the highlevel FST in case D drives stronger fluctuations and thus 'chaotic' vortical structures in the boundary layer, which are expected to cause much earlier transition as presented in figure $7(\mathrm{~d})$. The different states of the vortical structures obviously cause different paths of transition, and the details will be further discussed in $\S 4.2$.

\subsection{Breakdown mechanisms}

Affected by the structures formed at the leading-edge region and the continuing exposure to the FST, the suction-side boundary layers in the present simulations show differences in their transition behaviours, e.g. different transition onsets indicated by the wall-friction coefficient in figure $7(\mathrm{~d})$ and various structures shown in figure 8 . In this section, we will investigate the various transition mechanisms, including the instability of streaks in the cases with relatively low-level incoming turbulence, and the breakdown caused by vortical structures in the case with higher-level turbulence. 

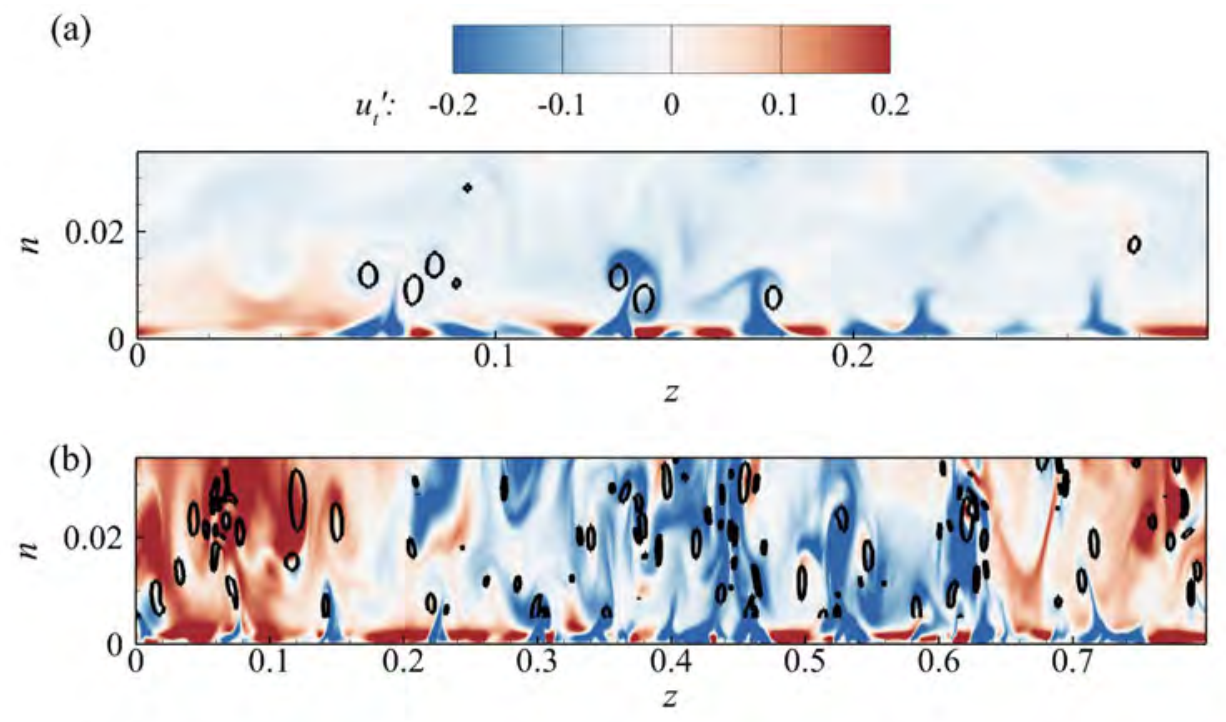

(c)
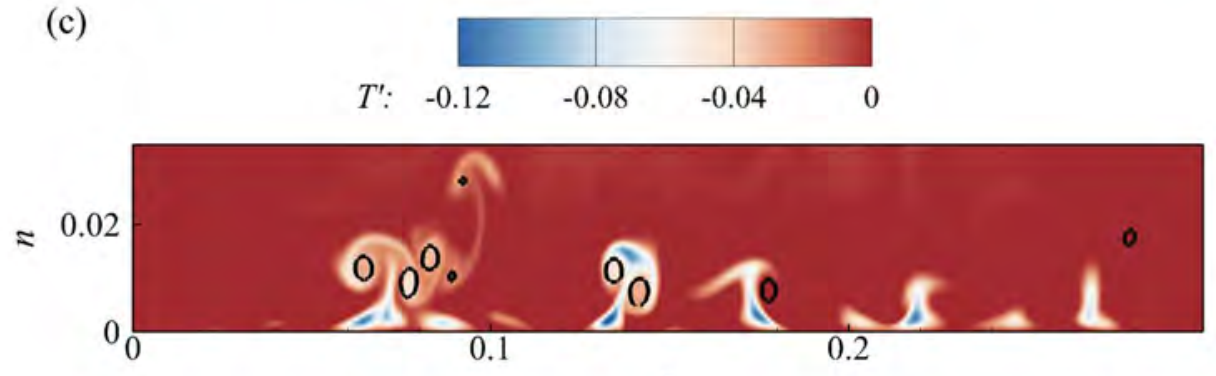

$z$
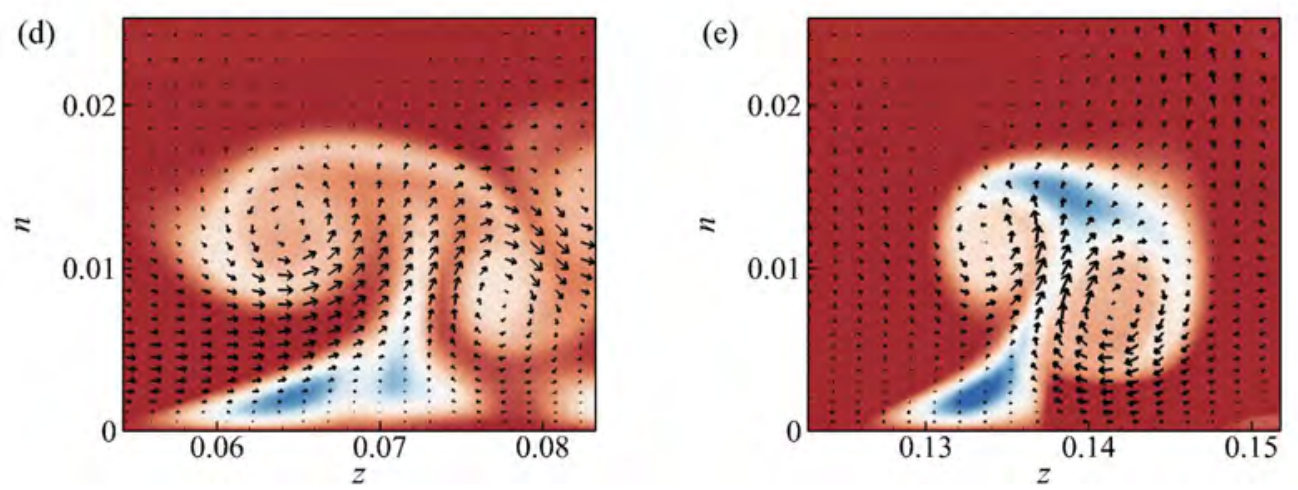

FIGURE 11. Instantaneous snapshots for contours of spanwise and wall-normal plane cuts at $x=0.3$ : (a) tangential velocity fluctuation $u_{t}^{\prime}$ for case $\mathrm{A}$; (b) $u_{t}^{\prime}$ for case $\mathrm{D}$; (c) temperature fluctuation $T^{\prime}$ for case A; (d) and (e): zoom-in views of 'mushroom-like' structures of $T^{\prime}$ with fluctuating velocity vectors. The iso-lines of $Q=4000$ are also presented in the contours. Note that the wall-normal coordinate $n$ is enlarged to clearly show the structures. 


\subsubsection{Varicose breakdown}

With the relatively low-level turbulence in cases A and B, the boundary layers show elongated streaks across the FPG region as presented in figures 8(a) and (b), and the transition onset is near the blade trailing edge under the APG. In figure 12, another view of the suction-side boundary layer for case A is given, displaying the contours of $u_{t}^{\prime}, u_{n}^{\prime}$ and $w^{\prime}$ on a surface at a distance of $\delta(x=0.5)$ from the wall. The low- and high-speed streaks in the streamwise direction can be shown by the $u_{t}^{\prime}$ contour. It is also apparent that for the streamwise streaks in the suction-side boundary layer, the tangential velocity fluctuation $u_{t}^{\prime}$ is much stronger than the other components. These streaks, which are usually considered as key features in bypass transition (Durbin \& Wu 2007), are closely related to the lift-up effects of the streamwise vortical structures formed at the leading edge (Andersson et al. 2001).

The instability of the streaks in the APG region, as highlighted by the red dashed circle in figure 12, is more clearly shown by the wall-normal and spanwise velocity fluctuations, and it is further studied in a zoom-in view in figure 13. Corresponding to the snapshot in figure 12 at $t=t_{0}$, the contour of $u_{n}^{\prime}$ in figure 13(a) shows a symmetric distribution about the streamwise direction. In addition, the iso-lines of $w^{\prime}$ are also presented, showing an antisymmetric pattern, with solid lines and dashed lines indicating $w^{\prime}=0.2$ and $w^{\prime}=-0.2$, respectively. It is noted that although the symmetry presented in figure 13(a) is not perfect due to the strong upstream turbulence, this type of instability corresponds well with varicose or symmetric modes (Andersson et al. 2001; Asai et al. 2002).

The inception of the varicose instability, which was found to be driven by the wallnormal shear of the streamwise flow by Skote et al. (2002), can be represented by the contours of $u_{t}^{\prime}$ on a cross-spanwise plane cut across the streak. In figure $14(\mathrm{a}) \sim(\mathrm{d})$, the snapshots are from a sequence of time instants at $t_{0}-2 \Delta t, t_{0}-\Delta t, t_{0}$, and $t_{0}+\Delta t$ respectively, with $t_{0}$ corresponding to the snapshot shown in figure 12 and $\Delta t=0.0115$. It is clearly shown that the varicose instability here happens due to the interactions between the low- and high-speed streaks (Lundell \& Alfredsson 2004; Brandt et al. 2004). Specifically, the fast-moving high-speed streak reaches the downstream low-speed region, and the instability is triggered at the interface in between. This instability grows rapidly at the tilting interface between the streaks, particularly with the presence of the APG which enhances the inflection for the streamwise velocity profiles. The development of the varicose mode instability can be further shown by the iso-surfaces of $u_{t}^{\prime}$ in figures $(\mathrm{e}) \sim(\mathrm{h})$, highlighting the formation of the vortical structures represented by the $Q$ isosurface. Convecting downstream, the streaks quickly break down as also suggested by the contours at two subsequent snapshots at $t_{0}+\Delta t$ and $t_{0}+2 \Delta t$ in figures $13(\mathrm{~b})$ and (c).

\subsubsection{Sinuous breakdown}

For the case $\mathrm{C}$ with higher-amplitude incoming turbulence, early-stage turbulent spots are observed in the FPG region, while the skin friction indicates that the mean flow stays laminar. In figure 15, a sequence of the contours of wall-normal velocity fluctuation $u_{n}^{\prime}$ is shown to track the formation of a turbulent spot, with the evolution of the spanwiseaveraged wall-friction coefficient $C_{f}$ for the corresponding snapshots also plotted on the suction-side surface. The contour surface is inside the boundary layer, at a distance of $\delta / 2$ away from the wall, where $\delta$ is the boundary layer thickness at $x=0.5$. In these snapshots, the spot precursor first stretches in the streamwise direction from figures 15 (a) to (b). As the amplitude of the disturbances is still small, the variation of the $C_{f}$ value near the spot is nearly negligible. Travelling downstream, the disturbances rapidly amplify and the precursor starts to break down, with the $C_{f}$ value around the spot significantly increasing 
(a)

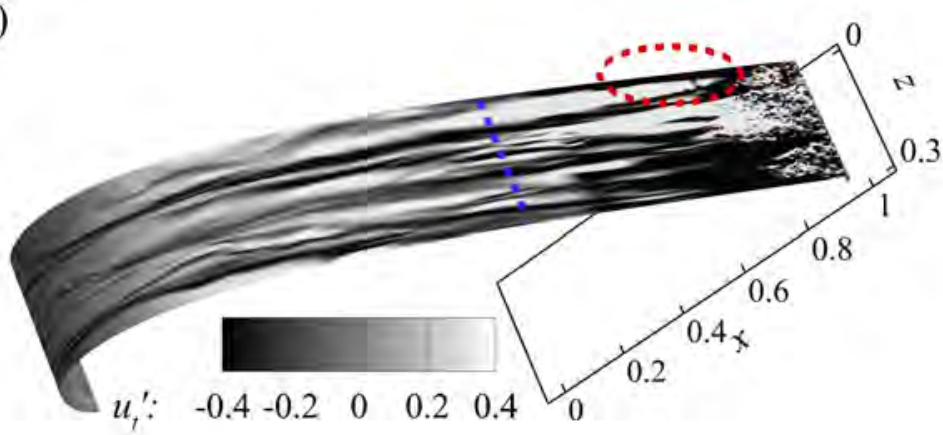

(b)

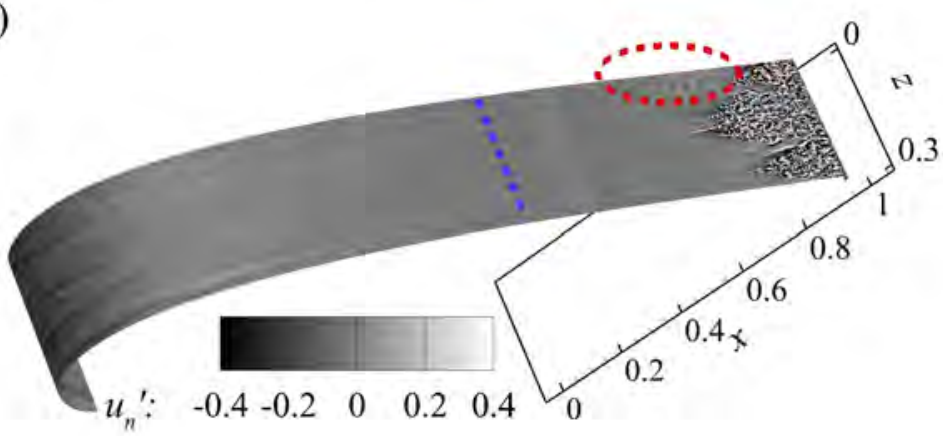

(c)

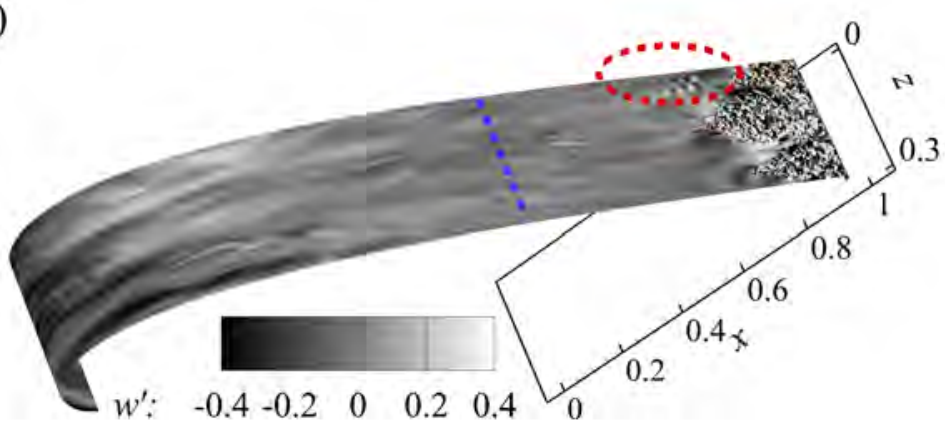

FiguRE 12. Instantaneous snapshots for streaks and the breakdown to turbulence at $t=t_{0}$ in case A. Contour of (a) streamwise component $u_{t}^{\prime}$, (b) wall-normal $u_{n}^{\prime}$, and (c) spanwise $w^{\prime}$ are presented at the surface which is $n=\frac{1}{2} \delta(x=0.5)$ from the wall. The blue dashed line indicates the boundary between FPG and APG at $x=0.8$, and an incipient turbulent spot is highlighted by the red dotted circle.

as shown in figure 15(c). Specifically, the breakdown of the spot precursor initiates at the tail and expands in the spanwise direction, resulting in a triangular shape, with the head towards the downstream direction. The spot continues to grow in both size and amplitude moving further downstream, which can be shown by the evolution of the $C_{f}$ wave-packet indicated by the blue arrow. Nevertheless, it is noted that the skin friction recovers to the undisturbed state again once the spot has passed by, suggesting that the disturbances for the formation and evolution of the turbulent spot are localized and there are calming effects trailing the spot. The temporal evolution of the displacement thickness of the boundary layer at $x=0.8$, which is indicated by the blue dashed line in 
(a)
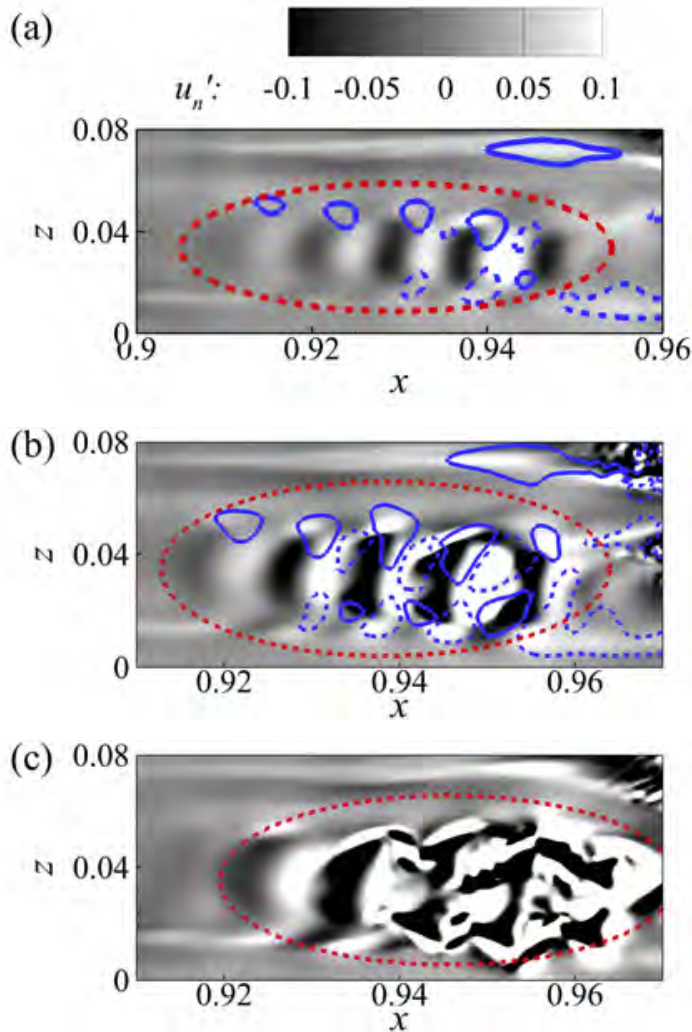

FiguRE 13. Zoom-in view of the instability of the streak in case A indicated by the red circle in figure 12. Instantaneous snapshots are presented at (a) $t=t_{0}$, (b) $t=t_{0}+0.0115$, and (c) $t=t_{0}+0.023$, with $t_{0}$ representing the snapshot shown in figure 12 . Contours of the wall-normal velocity fluctuation $u_{n}^{\prime}$ are presented on the surface $\delta / 2$ away from the wall, and the iso-lines of spanwise velocity fluctuation $w^{\prime}$ are also shown, with solid lines and dashed lines indicating $w^{\prime}=0.2$ and $w^{\prime}=-0.2$, respectively.

figure 15, is presented in figure 16, showing that the displacement thickness relaxes to a value even smaller than the undisturbed state after the spot-caused peak. As suggested by Katz et al. (1990), the low-displacement-thickness trailing the turbulent spot indicates the existence of a calmed region which is presumably caused by the distortion of the local mean flow by some nonlinear process. Finally, the enlarged spot merges with the turbulent boundary layer near the blade trailing edge, while a new spot precursor forms in the FPG region again as shown in figure 15(e).

In order to further investigate the incipient spot, a zoom-in view of the spot precursor is shown in figure 17, tracking the structures highlighted by the red dashed circle in the snapshots of figure 15(a), (b) and (c). Here, the vortical structures are identified by the iso-surface of $Q$ in the left figures, and the iso-surfaces of $u_{t}^{\prime}$ are also shown to track the development of the streamwise velocity fluctuation streaks. It is shown in figure 17(a) and (d) that there exists a quasi-streamwise vortex filament located in the strong spanwise shear region between the low- and high-speed streaks. The streamwise elongated vortex filament, which seems to be a remnant of the leading edge vortical structures, is tilting with the wall-normal vorticity component $\omega_{n}^{\prime} \sim \frac{\partial u_{t}^{\prime}}{\partial n}$. A secondary instability of the streak is then observed, caused by the strong spanwise shear in figure 17 (b) and (e), with the 

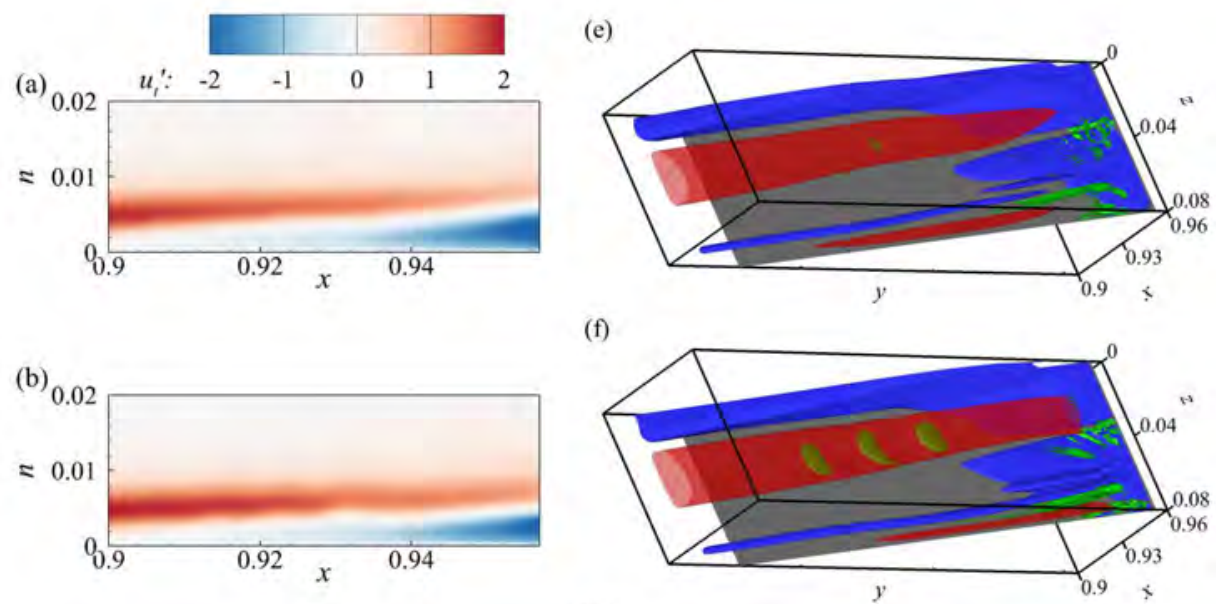

(g)
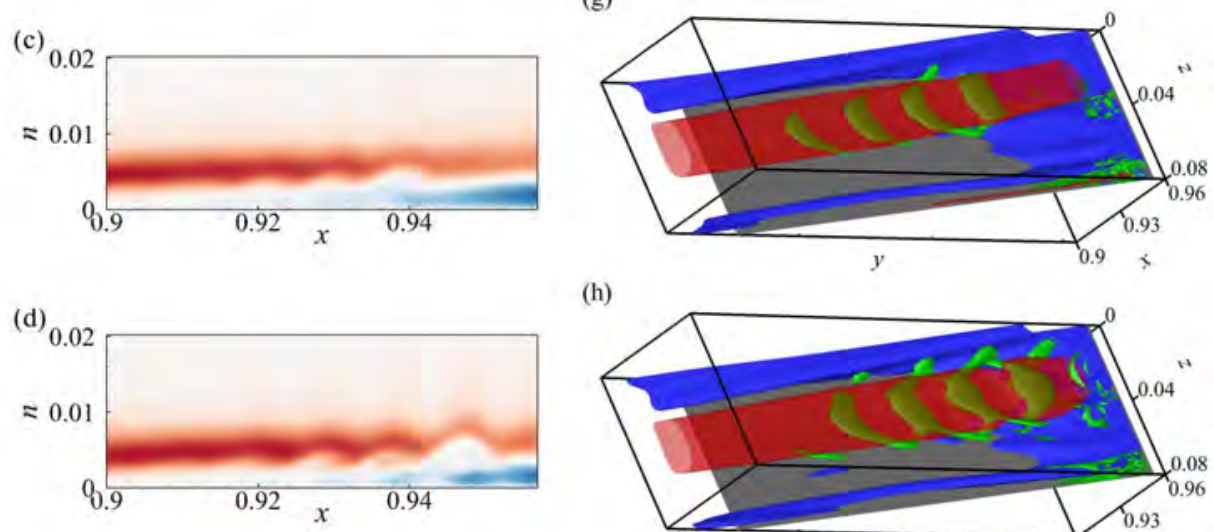

(h)

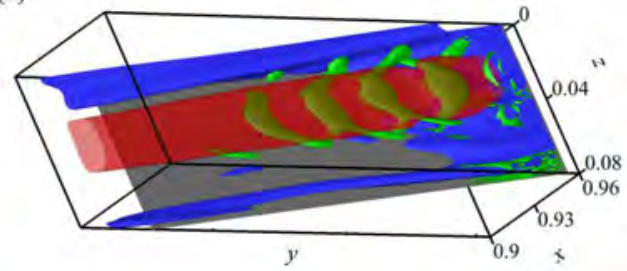

FiguRE 14. The inception of the varicose mode instability of the streak indicated by the red circle in figure 12. The contours of the tangential velocity perturbations $u_{t}^{\prime}$ on a cross-spanwise plane cut across the streak are shown by the instantaneous snapshots at (a) $t=t_{0}-0.023$, (b) $t=t_{0}-0.0115$, (c) $t=t_{0}$, and (d) $t=t_{0}+0.0115$, with $t_{0}$ representing the snapshot shown in figure 12. The iso-surfaces of $u_{t}^{\prime}=1.0$ (red), $u_{t}^{\prime}=-1.0$ (blue) and $Q=4000$ (green) are also shown at (e) $t=t_{0}-0.023$, (f) $t=t_{0}-0.0115$, (g) $t=t_{0}$, and (h) $t=t_{0}+0.0115$.

tail of the filament oscillating in the spanwise direction. Furthermore, the streak breaks down and forms the triangular spot seen in figure $17(\mathrm{c})$ and (f).

It is noted that the spanwise oscillations observed in figure $17(\mathrm{~b})$ are only on one side of the streak, which is in close agreement with the one-sided sinuous mode. As discussed by Brandt et al. (2004), the sinuous instability of low-speed streaks, though many are symmetric, can be dominated by single direction vorticity and oscillate only in one direction. This one-sided instability is believed to be related to the spanwise asymmetry of the streaks, which is indeed present for the case C with strong FST. Furthermore, contours of the streamwise velocity fluctuation $u_{t}^{\prime}$ in four cross-stream plane cuts at different streamwise positions, which correspond to the sinuous disturbances in figure 17(b), are shown in figure 18. Along with the vectors showing the fluctuating velocities in the wall-normal and spanwise directions, the meandering of the low-speed 
(a)

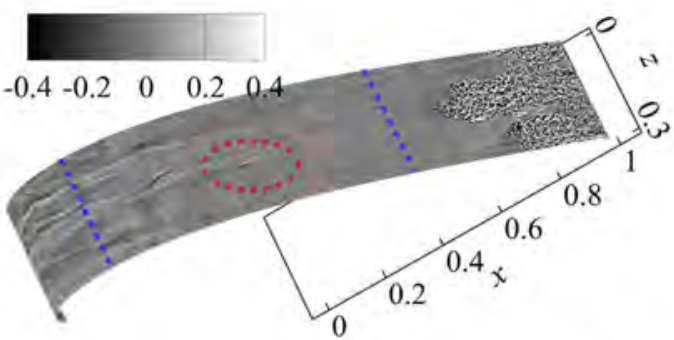

(b)

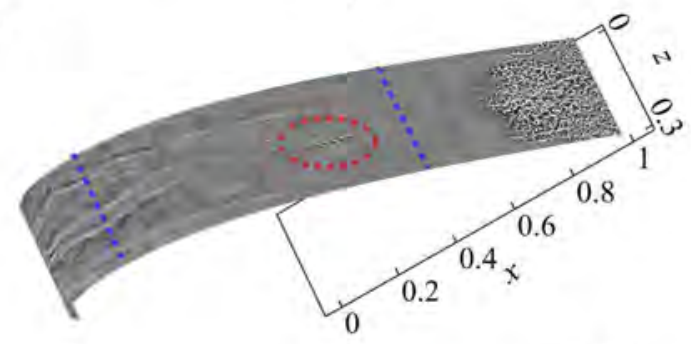

(c)

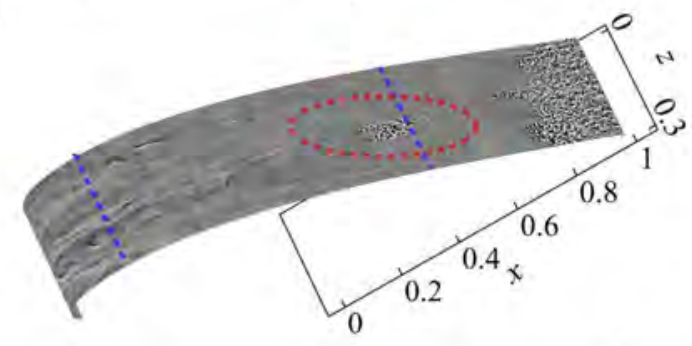

(d)

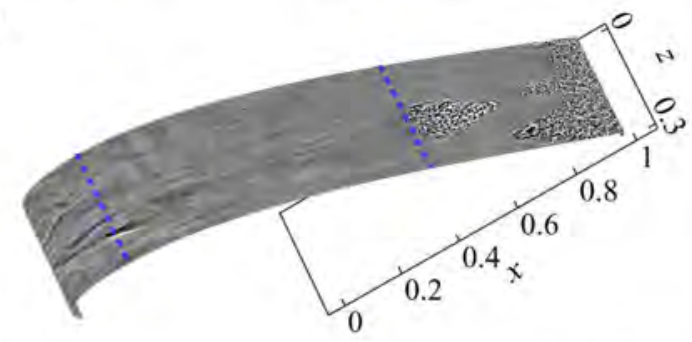

(e)

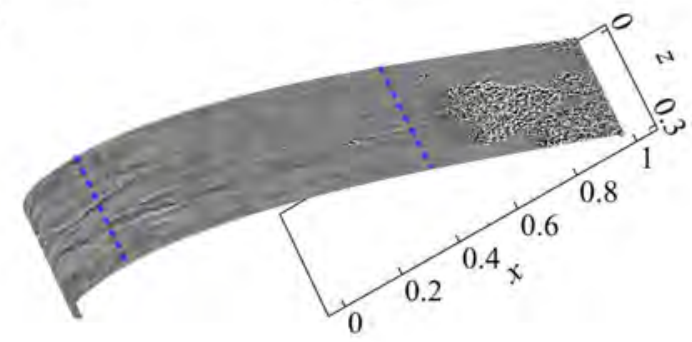

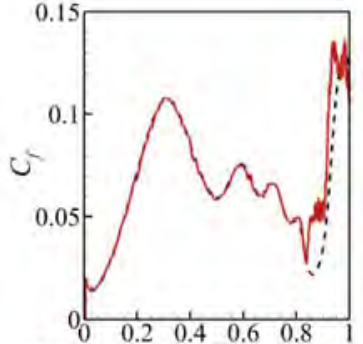
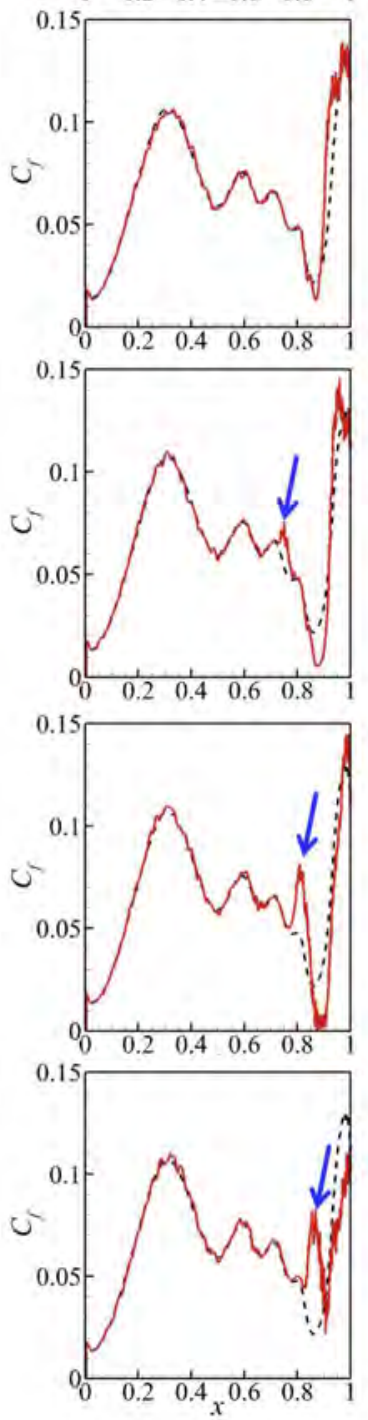

Figure 15. Formation of the turbulent spot in case C. Contours of wall-normal velocity fluctuation $u_{n}^{\prime}$ at the instantaneous snapshots: (a) $t=t_{0}$, (b) $t=t_{0}+0.069$, (c) $t=t_{0}+0.138$, (d) $t=t_{0}+0.207$, and (e) $t=t_{0}+0.276$. The blue dashed lines indicate $x=0.3$ and $x=0.8$, respectively, and the spot precursor is highlighted by the red dashed circle. Meanwhile, the wall-friction coefficient $C_{f}$ on the suction-side surface is averaged in the spanwise direction and plotted against $x$ for the corresponding snapshots (red solid lines), with the mean $C_{f}$ represented by the black dashed lines for reference. 


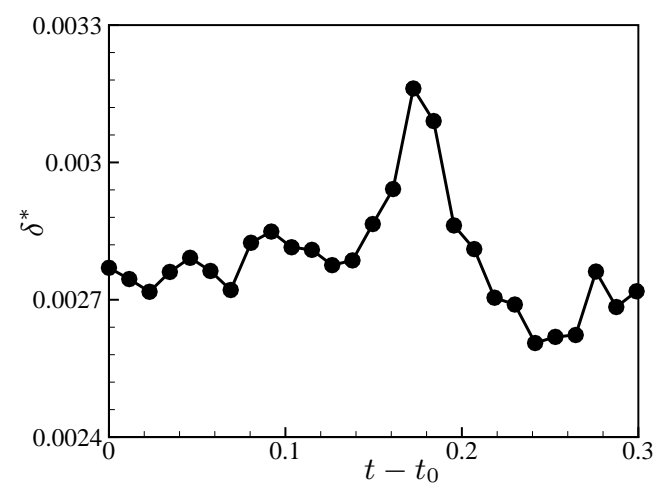

FiguRE 16. Temporal evolution of the displacement thickness of the boundary layer profile at $x=0.8$ in case $\mathrm{C}$. The instantaneous profile is spanwise averaged, starting from $t_{0}$ corresponding to figure $15(\mathrm{a})$.

(a)
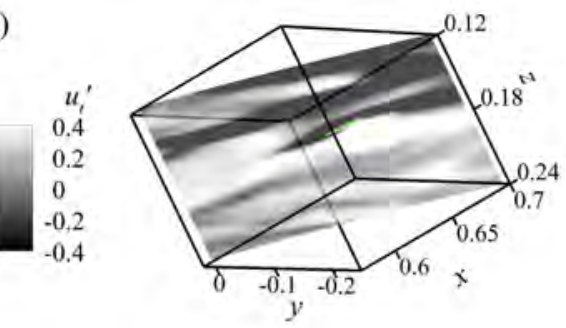

(b)

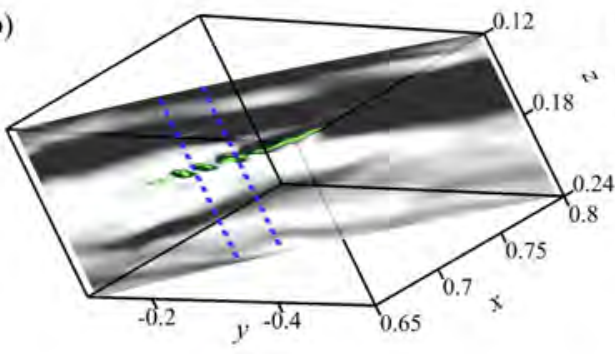

(c)

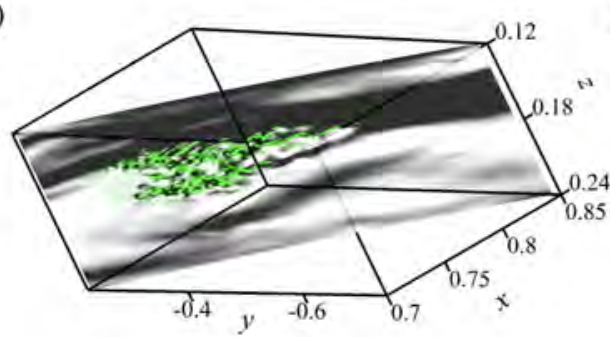

(d)

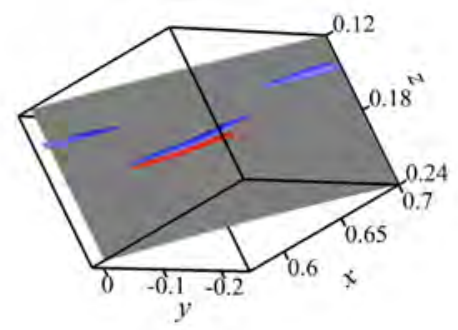

(e)

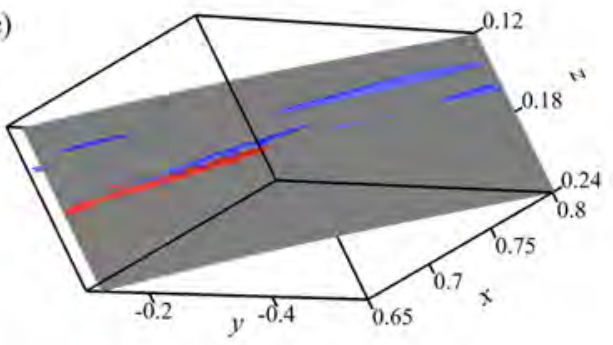

(f)

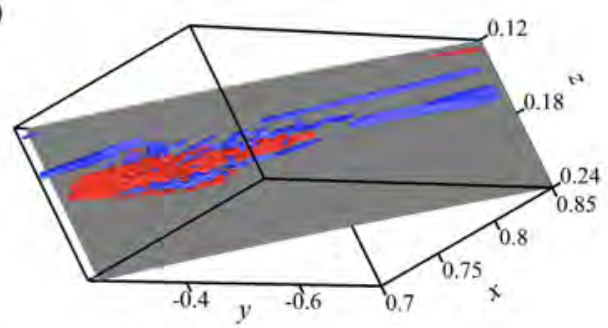

FIGURE 17. Zoom-in view of the instantaneous structures highlighted by the red-dashed circle in figure 15 to show the breakdown in case C. Contours of the streamwise velocity fluctuation $u_{t}^{\prime}$ is shown on the surface at $\delta / 2$ from the wall, and iso-surface of $Q=4000$ is also shown at (a) $t=t_{0}$, (b) $t=t_{0}+0.069$, (c) $t=t_{0}+0.138$. Iso-surfaces of $u_{t}^{\prime}=1.0$ (red) and $u_{t}^{\prime}=-1.0$ (blue) at (d) $t=t_{0}$, (e) $t=t_{0}+0.069$, (f) $t=t_{0}+0.138$. 

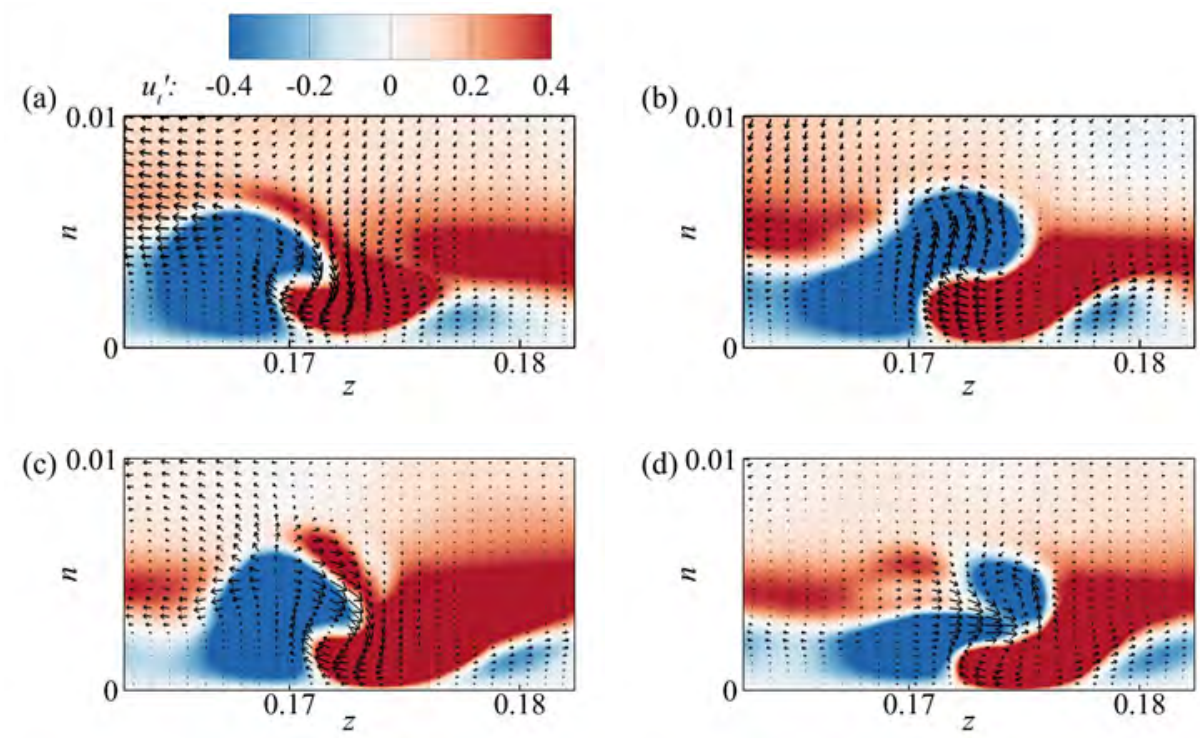

FIgURE 18. The inception of the varicose mode instability in case $\mathrm{C}$, corresponding to the snapshot shown in figure 17(b) at the instantaneous snapshot $t=t_{0}+0.069$. The contours of the tangential velocity perturbations $u_{t}^{\prime}$ are shown on four cross-streamwise plane cuts between the two blue dashed lines in figure $17(\mathrm{~b})$ at $x=0.70,0.705,0.71$, and 0.715 , and the vectors present the fluctuating velocities on the plane.

streak also gives further credibility to the hypothesis of the presence of a one-sided instability.

\subsubsection{Breakdown induced by vortex interaction}

For case D, the velocity fluctuations in the boundary layer are stronger than in other cases and the vortical structures are more 'chaotic' from the blade leading edge, as shown in figure 11. As a result, the transition onset shown by skin friction already occurs in the FPG region $(x<0.8)$ for case D, which is much earlier than in the other cases. In order to investigate the transition mechanisms, the breakdown process is presented by the contours of the wall-normal velocity fluctuations $u_{n}^{\prime}$ in figure 19. As a result of the largeamplitude inlet turbulence, the contours of $u_{n}^{\prime}$ for case D show much stronger fluctuations, compared to case $\mathrm{C}$ shown in figure 15 , that has the same turbulence amplitude at the inlet but a smaller length scale and thus exhibits more rapid decay of the incoming turbulence. In particular, once the flow enters into the region with relatively weak FPG and convex curvature, which is downstream of the first blue dashed line representing $x=0.5$, the fluctuations originating from the leading edge induce multiple structure breakdowns in the spanwise extent. One spot precursor, as highlighted by the red dashed circle in figure 19, is considered as an example and tracked through the formation process. The precursor is first observed just downstream of $x=0.5$, and it rapidly grows in size in both the streamwise and spanwise directions, travelling downstream. Thereafter, while the precursor breaks down to form a region packed with smaller-scale fluctuations, the shape of this region is obviously different from the spot shown in figure 15 .

The formation of the spot is further investigated in the zoom-in view presented in figure 20, with the vortical structures identified by the iso-surface of the $Q$-criterion and the contours of $u_{t}^{\prime}$ also shown. The instability of streaks, as presented for cases $\mathrm{A}, \mathrm{B}$ and $\mathrm{C}$ in $\S 4.2 .2$ and $\S 4.2 .1$, is not clearly observed here. Rather, the spot precursor 
(a)

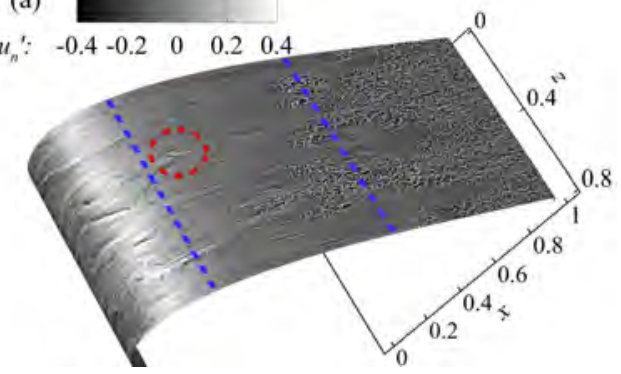

(c)

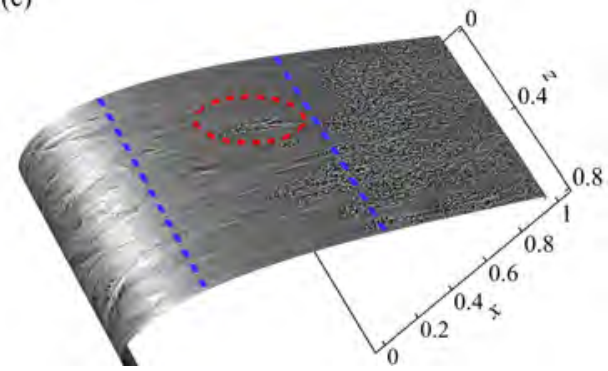

(b)

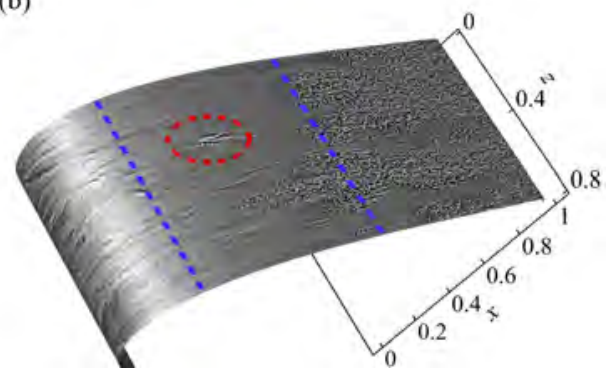

(d)

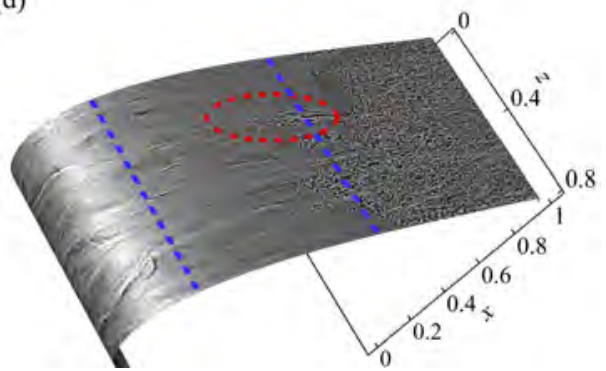

FIGURE 19. Formation of the turbulent spot in case D. Contours of wall-normal velocity fluctuation $u_{n}^{\prime}$ at the instantaneous snapshots: (a) $t=t_{0}$, (b) $t=t_{0}+0.046$, (c) $t=t_{0}+0.092$, and (d) $t=t_{0}+0.138$. The blue dashed lines indicate $x=0.5$ and $x=0.8$, respectively, and the spot precursor is highlighted by the red dashed circle.

(a)

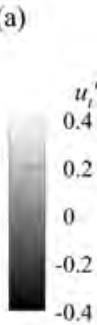

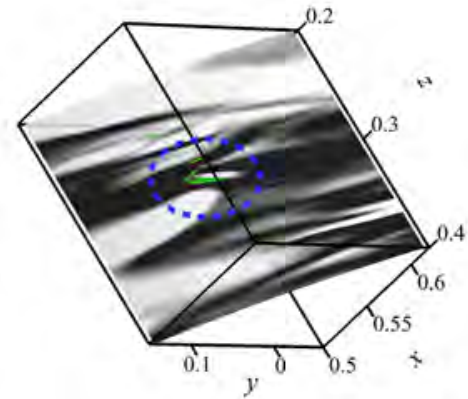

(b)

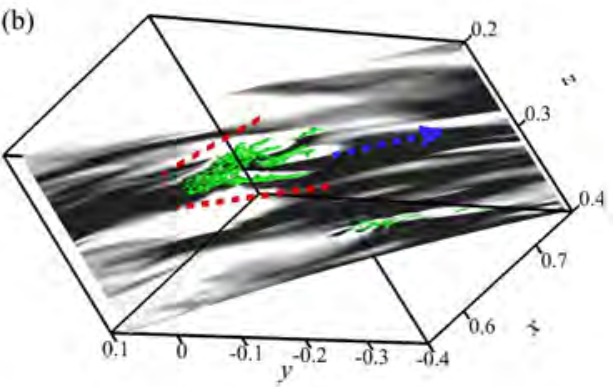

FiguRE 20. Zoom-in view of the instantaneous structures highlighted by the red dashed circle in figure 19 to show the breakdown in case $\mathrm{D}$, at: (a) $t=t_{0}$, and (b) $t=t_{0}+0.046$. Contours of the streamwise velocity fluctuation $u_{t}^{\prime}$ is shown on the surface at $\delta / 2$ from the wall, and iso-surfaces of $Q=4000$ is also shown.

results from the vortical breakdown. The pair of vortex filaments, as highlighted by the blue dashed circle in figure 20(a), forms a $\Lambda$-shape with the tip pointing in the negative streamwise direction, which is the opposite from the $\Lambda$-like structures usually observed in natural transition (Zhao et al. 2016a,b, 2018). The resulting spot, as presented in figure 20(b), is therefore different from the ordinary turbulent spots with the head towards the downstream direction, as shown in figure 17(c).

The breakdown of the precursor highlighted in figure 20(a) can be further shown by the contours of $u_{t}^{\prime}$ in the cross-spanwise plane cut at the tip of the precursor. In figure 21(a), the tip of the $\Lambda$-shaped vortex pair is located at the interface between the high- and low-speed region, as indicated by the black solid arrow. The vortex pair induces a downward movement of relatively high-speed fluid and elevates the upstream low-speed 


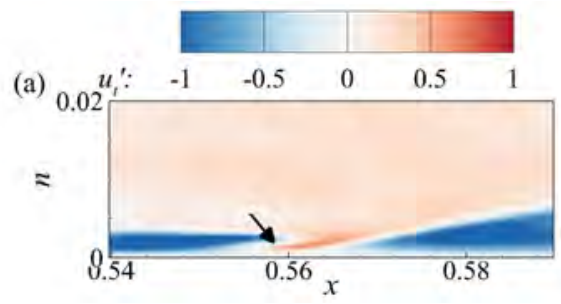

(b)
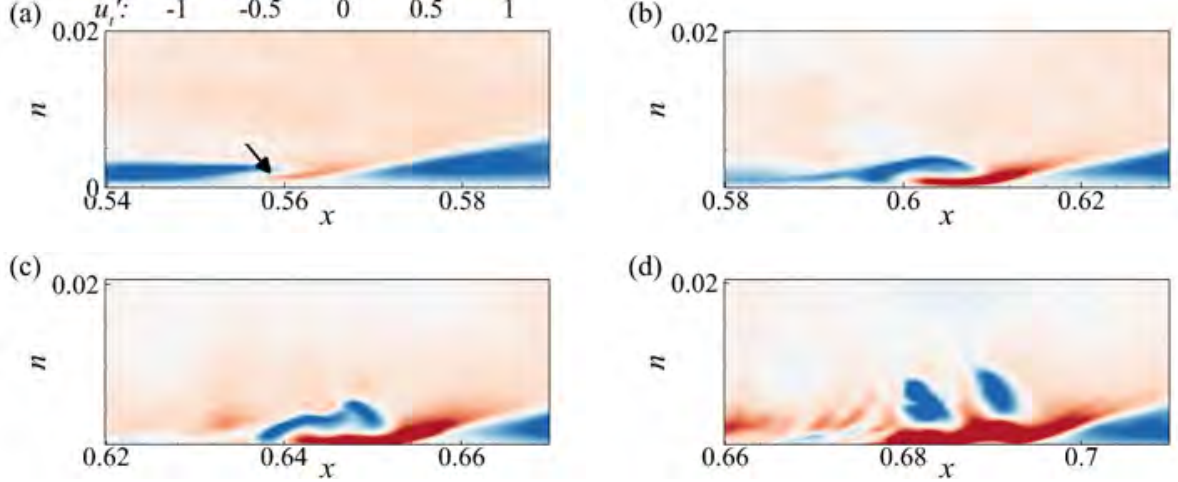

Figure 21. The incipient of the spot in case D. The contours of the instantaneous tangential velocity perturbations $u_{t}^{\prime}$ on a cross-spanwise plane cut at $z=0.29$ as indicated by the blue dashed arrow in figure 20: (a) $t=t_{0}$, (b) $t=t_{0}+0.023$, (c) $t=t_{0}+0.046$, and (d) $t=t_{0}+0.069$, with $t_{0}$ representing the snapshot shown in figure 20 (a). The window is moving at a speed of $c=1.74$ to track the spot precursor highlighted by the blue dashed circle in figure $20(\mathrm{a})$.

fluid from the wall, and the instability of the interface rapidly develops and then breaks down under the strong background turbulence, as shown in the subsequent snapshots in figures $21(\mathrm{~b}) \sim(\mathrm{d})$.

We remark here that the $\Lambda$-shaped vortex in figure $20(\mathrm{a})$ is just one example of the key structures. Due to the 'chaotic' nature of the vortical structures under the strong background turbulence, structures of different forms have also been identified in the formation of spot precursors in case D. The consistent mechanism for this type of breakdown, however, is that the precursors always originate from energetic vortical structures that can be traced back to the blade leading edge. The transition breakdown resulting from leading edge structures seems to be in close agreement with the leadingedge effects discussed in Nagarajan et al. (2007), in which the vortical effect is dominant when the bluntness is large and the FST is strong.

As a summary, the transition onsets in cases A and B are in the APG region close to the blade trailing edge. High- and low-speed streaks are observed, which are induced by the lift-up effects of the streamwise vortices formed at the leading edge. The structures then transform while convected downstream, due to a varicose mode instability that occurs as a result of the wall-normal inflectional instability under the APG, and which rapidly amplifies and causes the final breakdown to turbulence. For cases with stronger FST, however, the transition onsets occur much earlier. For case C, spot precursors form in the weak FPG region, and the formation process is through the secondary instability of streaks, which is observed to be the sinuous mode. Furthermore, with the even stronger FST entering the HPT cascade in case D, the vortical structures formed at the leading edge are in a chaotic manner and have a direct effect on the transition breakdown.

\section{Discussion on effects of incoming turbulence}

In this section, we will summarize the effects of the inlet turbulence on transition mechanisms in the present cases in which the suction side boundary layers are subject to pressure gradients and surface curvature, and the results will be compared to previous 
studies which have predominantly focused on flat-plate boundary layers with zeropressure gradient.

First of all, the effects of the turbulence length scales, in addition to the resulting different decay rate as presented in figure 4(a), are analyzed by comparing the transitional structures in cases A and B. It is noted that cases A and B have significantly different integral length scales of the incoming turbulence, as shown in figure 4(b), while the turbulence intensities upstream the leading edge $(x=-0.2)$ are relatively close to each other with $T_{u}=6.1 \% U_{i n}$ and $5.2 \% U_{i n}$, respectively. The transition onsets agree well with each other as observed in figure $7(\mathrm{~d})$, and streak-like structures and their instability are observed in both cases $\mathrm{A}$ and $\mathrm{B}$ as discussed in $\S 4.2 .1$. Therefore, a qualitative comparison between cases A and B implies that the transition mechanisms in relatively low-level FST cases are not directly influenced by the significant difference in integral length scales. Considering cases $\mathrm{C}$ and $\mathrm{D}$ which show distinctive levels of turbulence upstream the leading edge $\left(T_{u}=16.8 \% U_{i n}\right.$ and $9.5 \% U_{i n}$ at $\left.x=-0.2\right)$ and thus significantly different transitional behaviours as discussed in $\S 4$, the leading factor dominating the transition behaviours in the present cases seems to be whether the turbulence intensity is strong enough to overcome the stabilizing effect of the FPG.

However, a quantitative analysis is still needed to answer one open question - whether the length scales of the FST affect the scales of the transitional structures, especially the streamwise streaks in the boundary layer. In previous studies on bypass transition in flatplate boundary layers, the spanwise spacing of the streaks induced by FST was usually observed in experiments to be at the scale of the boundary layer thickness (Matsubara \& Alfredsson 2001), and it was reported in numerical simulations (Brandt et al. 2004) that the spanwise scale of the streaks is only weakly dependent on the scale of the disturbance in the free stream. Also, these are in accordance with the theoretical results in Luchini (2000) and Andersson et al. (1999), which show an optimal scale of 1.4 times boundary layer thickness on a flat surface. On the other hand, Ovchinnikov et al. (2008) reported that the FST length scale can affect not only the onset of transition, but also the underlying physical mechanism including the critical vortical structures and spacing of the streaks. More recently, the importance of the integral length scales on predicting transition onset is stressed based on the experimental data consisting of 42 FST states (Fransson 2017).

In the present study, the spanwise spacings of the streaks in cases A and B, quantified by twice the distance to the minimum of the spanwise correlations of $u_{t}^{\prime}$ shown in figure 22(a), are compared with the local boundary layer thickness. As becomes evident from figure 22(b), the spanwise spacings at the suction-peak $x=0.3$ in cases $\mathrm{A}$ and B are $\Delta_{z}=0.058$ and 0.18 , respectively, which are of the order of the integral length scales from the inlet turbulence and much larger than the boundary layer thickness. Furthermore, the spanwise spacings of the streaks slightly increase in the FPG boundary layer, corresponding to the growth of the streaks in both size and intensity. We thus conclude here that the spanwise spacings of the streaks in the present study depend on the FST and are not directly connected to the boundary layer thickness. It is also interesting to compare the present streaks in the transitional boundary layers with the well-known streaks as key coherent structures in fully turbulent boundary layers. The spanwise spacing of wall-turbulence streaks was found exhibiting consistent values of $\Delta_{z}^{+} \approx 100$ in the near-wall region (Smith \& Metzler 1983) and can go as high as $\Delta_{z}^{+} \approx 1500$ in the outer layer (Jiménez 2018), while the spanwise spacings of the streaks in the present study, normalized in wall-units, are $\Delta_{z}^{+} \approx 1000 \sim 1500$ in case A and $\Delta_{z}^{+} \approx 3000 \sim 4000$ in case B. Moreover, Brinkerhoff \& Yaras (2015) found in a numerical simulation of flatplate boundary layer with pressure gradient that although the stabilizing effects of a 

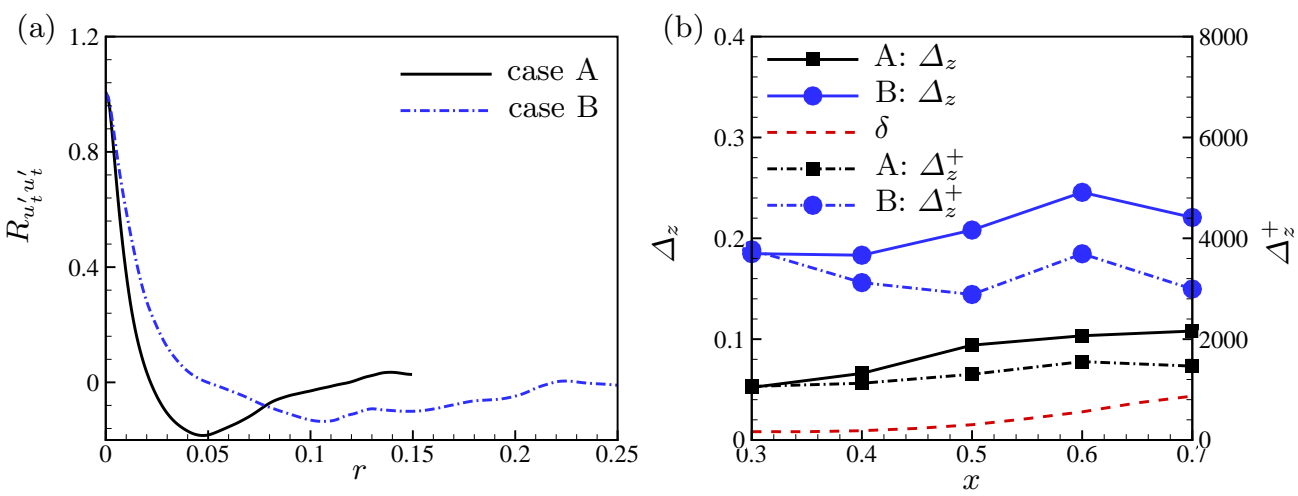

Figure 22. (a) Spanwise correlation of the tangential velocity fluctuation $u_{t}^{\prime}$ on the suction-side boundary layer at $x=0.5$. (b) Development of the spanwise spacing of the streaks $\Delta_{z}$ compared to the local boundary layer thickness $\delta$, and spacing normalized in the local wall units $\Delta_{z}^{+}$.

favourable pressure gradient can cause a larger streak spacing in wall units, resulting in typical spacing of $\Delta_{z}^{+} \approx 198$, the streak spacing seems to be at the scale of the local boundary layer thickness with the existence of acceleration. Therefore, considering the much larger streak spacing compared to the local boundary layer thickness found in the current work, and the significant variations of streak spacings in cases A and B, it is likely that the streaks here mainly originate from the vortical structures formed at the blunt blade leading edge as a consequence of the incoming turbulence, rather than through a scale-selection of the boundary layer.

The development of the fluctuations in the boundary layer can be characterized by the wall-normal maximum of the tangential velocity fluctuation $\max _{n}\left(u_{t}^{\prime}\right)^{2}$. As shown in figure 23(a), $\max _{n}\left(u_{t}^{\prime}\right)^{2}$ in case A first decreases due to the large-amplitude curvature and the strong pressure gradient, as indicated in figures $7(\mathrm{~b})$ and (c). Downstream of $x=0.5$, the curvature effect and the FPG turn much weaker, and the streamwise streaks start growing. As a comparison, figure 23(b) shows that the spanwise component $\max _{n}\left(w^{\prime}\right)^{2}$ monotonously decreases until the transition onset, after which the growth rate of $\max _{n}\left(u_{t}^{\prime}\right)^{2}$ also significantly increases. This suggests that the streamwise streaks in the boundary layer are dominated by the fluctuations in the streamwise direction, and the strength of the streaks keeps increasing for the weak FPG and APG regions.

In addition, the effects of the inlet turbulence can be studied by comparing results between all the cases. It is shown that compared to cases A, B and C, the growth rate of $\max _{n}\left(u_{t}^{\prime}\right)^{2}$ in case $\mathrm{D}$ is much larger and the spanwise fluctuations are apparently much stronger, which is in accordance with the structures being more chaotic and consistent with the fact that a different path from streak instability is responsible for the transition. Furthermore, for the cases $\mathrm{A}, \mathrm{B}$ and $\mathrm{C}$ which show obvious streak instabilities during transition, the growth rates in the weakly FPG region (from $x=0.5$ to $x=0.8$ ) are compared in figure $23(\mathrm{c})$, showing that $\max _{n}\left(u_{t}^{\prime}\right)^{2}$ grows approximately linearly with the Reynolds number based on the surface distance $R e_{s}$. This observation agrees with previous experimental (Kendall 1985; Matsubara \& Alfredsson 2001) and theoretical results (Andersson et al. 1999; Luchini 2000) obtained for flat plate boundary layers with zero-pressure gradient. Furthermore, the algebraic growth of streaks was also observed in the flat-plate boundary layer simulation with pressure gradient by Brinkerhoff \& Yaras (2015), in which the variation of favourable pressure gradient seems to not affect the growth rate. The differences observed in the present cases compared to the flat-plate 

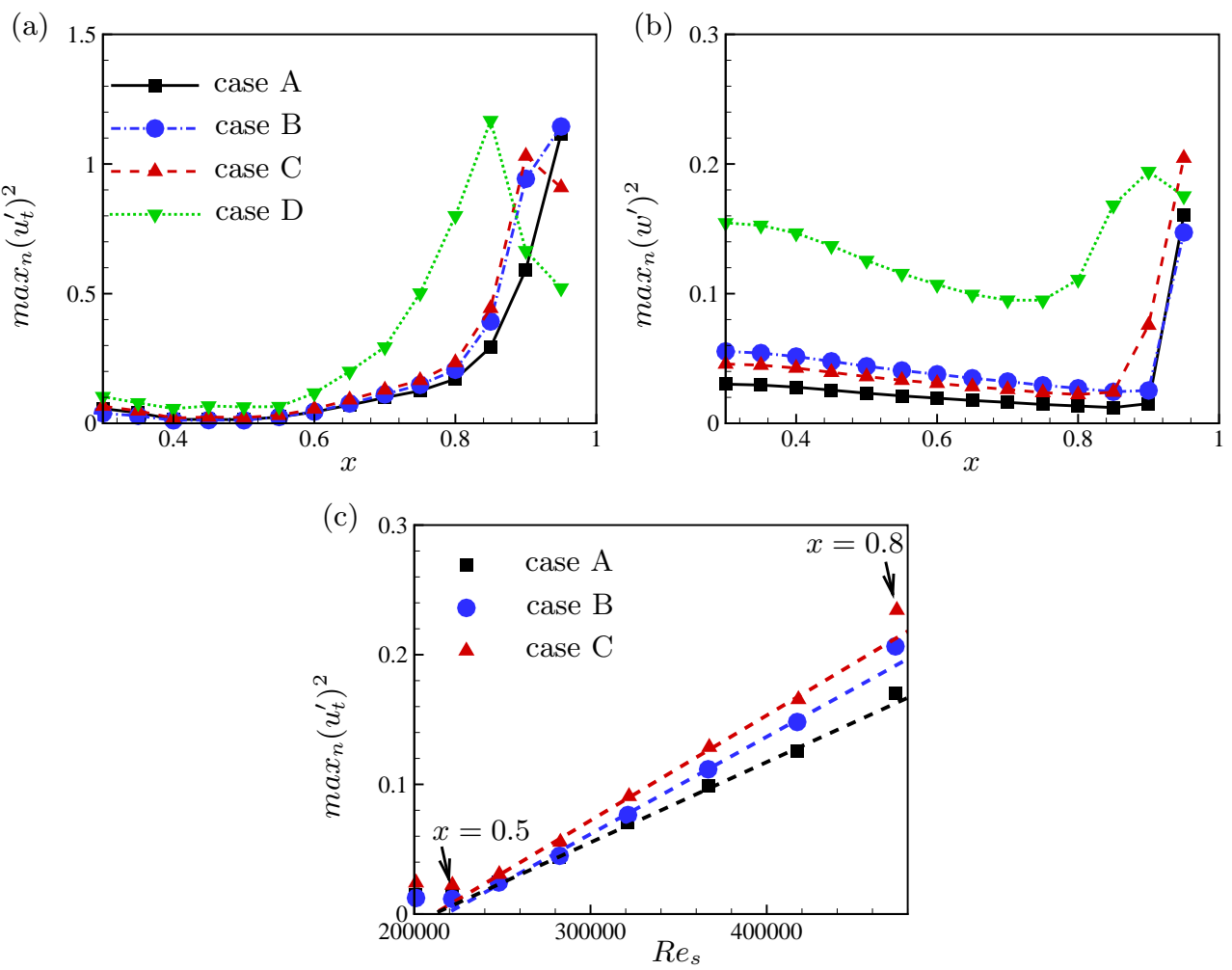

FIGURE 23. Development of the wall-normal maximum of the streamwise velocity fluctuation.

boundary layer in Brinkerhoff \& Yaras (2015), especially the strong stabilizing effects causing the decrease of the streak intensity at $x<0.5$ as shown in figure 23(a), highlight the influence of the strong FPG and convex curvature by the blunt leading edge. To summarize, even though the streamwise streaks in the present cases are mainly induced by the vortical structures formed near the leading edge region, and thus have spanwise scales much larger than the local boundary layer thickness, the growth of the streaks at the downstream stage with weak pressure gradient and curvature are still closely related to the algebraic or transient growth theory. Moreover, it is shown that the growth rates of $\max _{n}\left(u_{t}^{\prime}\right)^{2}$ in cases with stronger FST are slightly larger, implying that the growth of the streaks is also affected by the FST (Asai et al. 2007).

The development of the velocity fluctuations along the suction-side boundary layer can be further characterized by the spanwise spectra, as presented in figure 24. The spectra in the streamwise $\hat{u}_{t}^{\prime}$, the wall-normal $\hat{u}_{n}^{\prime}$, and the spanwise directions $\hat{w}^{\prime}$ are obtained as

$$
\hat{u}_{i}^{\prime}\left(x, k_{z}\right)=\mathcal{F}_{z}\left\{u_{i}^{\prime}(x, z)\right\},
$$

where $\mathcal{F}_{z}$ represents the Fourier transform in the spanwise direction. In figure 24, the time-averaged spectra $\left\langle\left|\hat{u}_{i}^{\prime}\left(x, k_{z}\right)\right|^{2}\right\rangle$ for case $\mathrm{A}$ are plotted against $x$ at the surface which is $\frac{1}{2} \delta(x=0.5)$ from the wall, and the case $\mathrm{D}$ with much higher levels and greater length scales of FST is presented for comparison. It is apparent that the velocity fluctuations are mainly at low wavenumbers before the boundary layer transitions into turbulence, which suggests the boundary layer is predominately affected by the large-scale part of the FST. After transition, the high-wavenumber amplitudes for all of the components significantly amplify, and the spectra become broadband. Furthermore, the streamwise 
fluctuating velocity is shown to be dominant for most of the boundary layer, while the wall-normal component $\left\langle\left|\hat{u}_{n}^{\prime}\left(x, k_{z}\right)\right|^{2}\right\rangle$ is negligible, until transition to turbulence has occurred. The spanwise component $\left\langle\left|\hat{w}^{\prime}\left(x, k_{z}\right)\right|^{2}\right\rangle$, however, is prevailing in the LE region, but the amplitude gradually decreases in the FPG region $(x<0.8)$. This is presumably due to the stabilizing effects of the FPG and the convex surface. Compared to case A, the transition in case $\mathrm{D}$ takes place much earlier due to the higher-amplitude FST. This is also shown in figures $8(\mathrm{~d}) \sim(\mathrm{f})$, in which the spectra of fluctuating velocities in case $\mathrm{D}$ turn to broadband at around $x=0.8$.

In addition, it is worth noting that one peak of the $\left\langle\left|\hat{u}_{t}^{\prime}\left(x, k_{z}\right)\right|^{2}\right\rangle$ spectrum in the LE region is shown by the red arrow in figure $24(\mathrm{a})$, which indicates a wave length of

$$
\lambda_{z}=\frac{2 \pi}{k_{z}} \approx 0.058 \text {. }
$$

This wave length corresponds to the spanwise spacing of the low-speed streaks at the leading-edge region $x=0.3$ in case $\mathrm{A}$, as also observed in figure $22(\mathrm{~b})$.

\section{Conclusions}

In the present study, large-eddy simulations of a high-pressure turbine at realistic Reynolds and Mach numbers have been performed, and the inlet turbulence was varied in four cases to study the effects of turbulence intensities and length scales on the boundary layer transition. Due to the interactions between the incoming turbulence and the blunt blade leading edge, vortical structures wrap around the leading edge and thus affect the boundary layer transition on the suction-side. For cases with different FST, various paths for transition are observed, including the sinuous and varicose modes of streak instability and the breakdown caused by vortical interactions.

In cases with relatively low-level turbulence, i.e. cases $\mathrm{A}, \mathrm{B}$ and $\mathrm{C}$, the low- and highspeed streaks are observed to originate from the leading-edge vortical structures, and the spanwise length scales of the streaks are related to the integral length scales of the upstream turbulence. While the streaks are mainly induced by the leading edge vortical structures and subject to favorable pressure gradient and curvature effects, the boundary layer fluctuations grow linearly with $R e_{s}$, which is in close agreement with the algebraic or transient growth originally proposed in flat plate boundary layer with zero-pressure gradient. Furthermore, the streaks break down and the transition onsets are through the instability of the streaks, either the varicose mode in the APG region or the sinuous mode earlier in the weak FPG region. For cases with higher levels of FST, however, the leading-edge structures are more chaotic and no obvious streak instability is observed. Accordingly, the transition onset occurs much earlier on the suction-side, through a breakdown caused by interactions between vortical structures.

This research used resources of the Oak Ridge Leadership Computing Facility, which is a DOE Office of Science User Facility supported under Contract DE-AC05-00OR22725. This work was also supported by the resources provided by the Pawsey Supercomputing Centre with funding from the Australian Government and the Government of Western Australia. This work was also supported by a grant from the Swiss National Supercomputing Centre (CSCS) under project ID s884.

\footnotetext{
Declaration of Interests. The authors report no conflict of interest.
} 

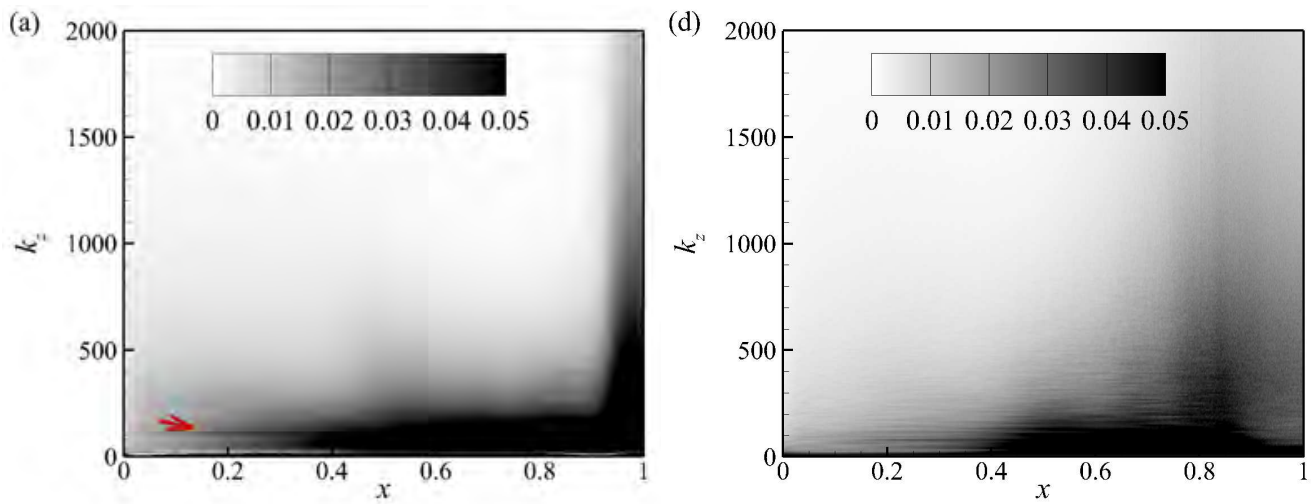

(b)

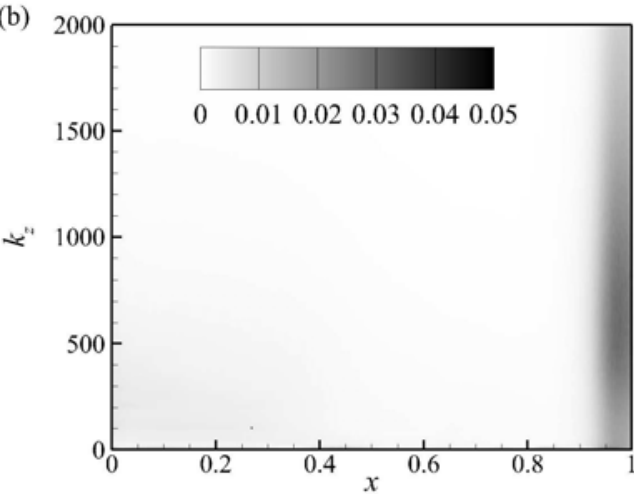

(c)

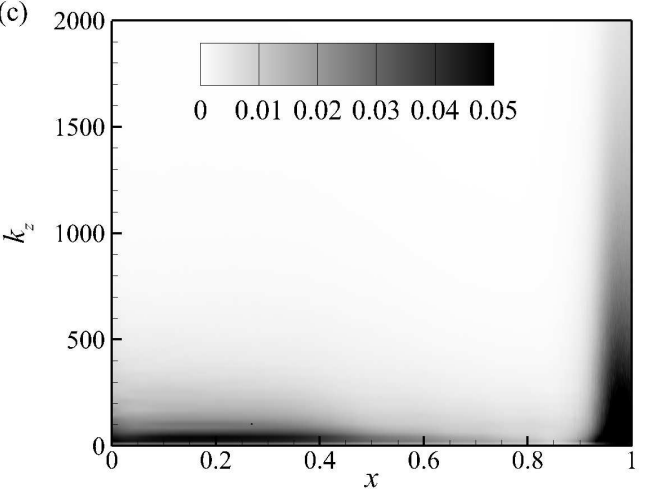

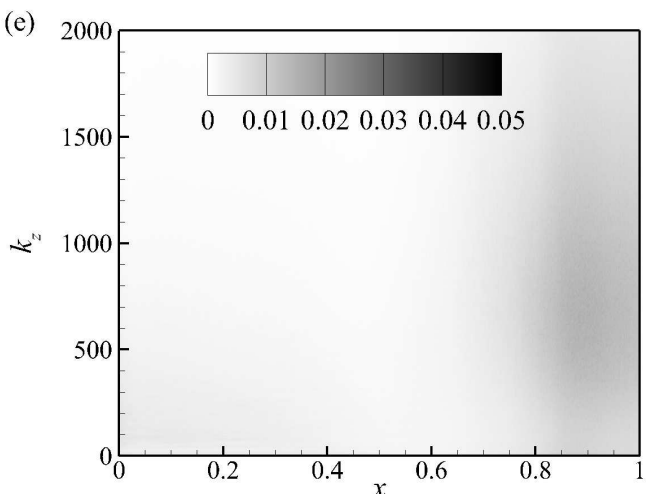

(f)

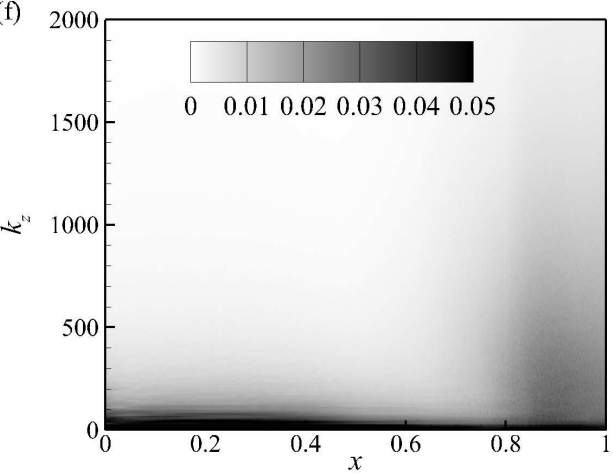

FIGURE 24. The evolution of the spanwise spectra of the fluctuating velocities along the suction-side blade. The spectra of the velocity components are extracted at the surface which is $n=\frac{1}{2} \delta(x=0.5)$ from the wall and plotted against the streamwise coordinate $x$ and spanwise wavenumber $k_{z}$. The contours are (a) $\left\langle\left|\hat{u}_{t}^{\prime}\left(x, k_{z}\right)\right|^{2}\right\rangle$ in case A; (b) $\left\langle\left|\hat{u}_{n}^{\prime}\left(x, k_{z}\right)\right|^{2}\right\rangle$ in case A; (c) $\left\langle\left|\hat{w}^{\prime}\left(x, k_{z}\right)\right|^{2}\right\rangle$ in case A; (d) $\left\langle\left|\hat{u}_{t}^{\prime}\left(x, k_{z}\right)\right|^{2}\right\rangle$ in case D; (e) $\left\langle\left|\hat{u}_{n}^{\prime}\left(x, k_{z}\right)\right|^{2}\right\rangle$ in case D; (f) $\left\langle\left|\hat{w}^{\prime}\left(x, k_{z}\right)\right|^{2}\right\rangle$ in case D.

\section{REFERENCES}

Abu-Ghannam, B. J. \& Shaw, R. 1980 Natural transition of boundary layersthe effects of turbulence, pressure gradient, and flow history. J. Mech. Engng Sci. 22 (5), 213-228.

Alam, M. \& Sandham, N. D. 2000 Direct numerical simulation of short laminar separation bubbles with turbulent reattachment. J. Fluid Mech. 410, 1-28. 
Andersson, P., Berggren, M. \& Henningson, D. S. 1999 Optimal disturbances and bypass transition in boundary layers. Phys. Fluids 11 (1), 134-150.

Andersson, P., Brandt, L., Bottaro, A. \& Henningson, D. S. 2001 On the breakdown of boundary layer streaks. J. Fluid Mech. 428, 29-60.

Araya, G., Castillo, L. \& Hussain, F. 2015 The log behaviour of the reynolds shear stress in accelerating turbulent boundary layers. J. Fluid Mech. 775, 189-200.

Arts, T., Lambertderouvroit, M. \& Rutherford, A. W. 1990 Aero-thermal investigation of a highly loaded transonic linear turbine guide vane cascade. A test case for inviscid and viscous flow computations. NASA STI/Recon Technical Report N 91.

Asai, M., Konishi, Y., Oizumi, Y. \& Nishioka, M. 2007 Growth and breakdown of low-speed streaks leading to wall turbulence. J. Fluid Mech. 586, 371-396.

Asai, M., Minagawa, M. \& NishiokA, M. 2002 The instability and breakdown of a near-wall low-speed streak. J. Fluid Mech. 455, 289-314.

Bhaskaran, R. \& Lele, S. K. 2010 Large eddy simulation of free-stream turbulence effects on heat transfer to a high-pressure turbine cascade. J. Turbul. 11 (6), 1-15.

BRANDT, L. 2014 The lift-up effect: the linear mechanism behind transition and turbulence in shear flows. Eur. J. Mech. B-Fluid 47, 80-96.

Brandt, L., Schlatter, P. \& Henningson, D. S. 2004 Transition in boundary layers subject to free-stream turbulence. J. Fluid Mech. 517, 167-198.

Brinkerhoff, J. R. \& YARAS, M. I. 2015 Numerical investigation of transition in a boundary layer subjected to favourable and adverse streamwise pressure gradients and elevated free stream turbulence. J. Fluid Mech. 781, 52-86.

Durbin, P. A. \& Wu, X. 2007 Transition beneath vortical disturbances. Annu. Rev. Fluid Mech. 39, 107-128.

FrANSSON, J. H. M. 2017 Free-stream turbulence and its influence on boundary-layer transition. In 10th International Symposium on Turbulence and Shear Flow Phenomena, pp. 6D-5.

Goldstein, M. E. \& Wundrow, D. W. 1998 On the environmental realizability of algebraically growing disturbances and their relation to klebanoff modes. Theor. Comput. Fluid Dyn. 10 (1-4), 171-186.

Gostelow, J. P., Blunden, A. R. \& Walker, G. J. 1992 Effects of free-stream turbulence and adverse pressure gradients on boundary layer transition. J. Turbomach. 116, 392404.

Herbert, T. 1984 Analysis of the subharmonic route to transition in boundary-layers. AIAA Paper 84, 0009.

Herbert, T. 1988 Secondary instability of boundary layers. Annu. Rev. Fluid Mech. 20, 487526.

Hunt, J. C. R., Wray, A. A. \& Moin, P. 1988 Eddies, streams, and convergence zones in turbulent flows. In Studying turbulence using numerical simulation databases, 2, , vol. 1, pp. $193-208$.

Jacobs, R. G. \& Durbin, P. A. 1998 Shear sheltering and the continuous spectrum of the Orr-Sommerfeld equation. Phys. Fluids 10 (8), 2006-2011.

Jacobs, R. G. \& Durbin, P. A. 2001 Simulations of bypass transition. J. Fluid Mech. 428, $185-212$.

JimÉNEZ, J. 2018 Coherent structures in wall-bounded turbulence. J. Fluid Mech. 842.

Jones, L. E., Sandberg, R. D. \& Sandham, N. D. 2008 Direct numerical simulations of forced and unforced separation bubbles on an airfoil at incidence. J. Fluid Mech. 602, $175-207$.

Katz, Y., Seifert, A. \& Wygnanski, I. 1990 On the evolution of the turbulent spot in a laminar boundary layer with a favourable pressure gradient. J. Fluid Mech. 221, 1-22.

Kendall, J. 1985 Experimental study of disturbances produced in a pre-transitional laminar boundary layer by weak freestream turbulence. AIAA Paper pp. 85-1695.

Kendall, J. M. 1991 Studies on laminar boundary-layer receptivity to freestream turbulence near a leading edge. In Boundary layer stability and transition to turbulence (ed. D. C. Reda, H. L. Reed \& R. Kobayashi), pp. 23-30. ASME.

Kennedy, C. A., Carpenter, M. H. \& Lewis, R. M. 2000 Low-storage, explicit RungeKutta schemes for the compressible Navier-Stokes equations. Appl. Numer. Math. 35 (3), $177-219$.

Khapko, T., Kreilos, T., Schlatter, P., Duguet, Y., Eckhardt, B. \& Henningson, 
D. S. 2016 Edge states as mediators of bypass transition in boundary-layer flows. J. Fluid Mech. 801.

KIM, J. W. \& LeE, D. J. 2003 Characteristic interface conditions for multiblock high-order computation on singular structured grid. AIAA J. 41 (12), 2341-2348.

Kim, J. W. \& SAndBerg, R. D. 2012 Efficient parallel computing with a compact finite difference scheme. Comput. Fluids 58, 70-87.

Klebanoff, P. S., Tidstrom, K. D. \& Sargent, L. M. 1962 The three-dimensional nature of boundary-layer instability. J. Fluid Mech. 12, 1-34.

Klein, M., SAdiki, A. \& JAnickA, J. 2003 A digital filter based generation of inflow data for spatially developing direct numerical or large eddy simulations. J. Comp. Phys. 186 (2), $652-665$.

Kreilos, T., Khapko, T., Schlatter, P., Duguet, Y., Henningson, D. S. \& Eckhardt, B. 2016 Bypass transition and spot nucleation in boundary layers. Phys. Rev. Fluids 1 (4), 043602.

Luchini, P. 2000 Reynolds-number-independent instability of the boundary layer over a flat surface: optimal perturbations. J. Fluid Mech. 404, 289-309.

Lundell, F. \& Alfredsson, P. H. 2004 Streamwise scaling of streaks in laminar boundary layers subjected to free-stream turbulence. Phys. Fluids 16 (5), 1814-1817.

Mandal, A. C., Venkatakrishnan, L. \& Dey, J. 2010 A study on boundary-layer transition induced by free-stream turbulence. J. Fluid Mech. 660, 114-146.

Marxen, O. \& ZaKi, T. A. 2019 Turbulence in intermittent transitional boundary layers and in turbulence spots. J. Fluid Mech. 860, 350-383.

Matsubara, M. \& Alfredsson, P. H. 2001 Disturbance growth in boundary layers subjected to free-stream turbulence. J. Fluid Mech. 430, 149-168.

Michelassi, V., Wissink, J. \& Rodi, W. 2002 Analysis of DNS and LES of flow in a low pressure turbine cascade with incoming wakes and comparison with experiments. Flow Turbul. Combust. 69 (3-4), 295-329.

Morkovin, M. V. 1969 On the many faces of transition. In Viscous drag reduction (ed. C. S. Wells), pp. 1-31. Springer.

Muck, K. C., Hoffmann, P. H. \& Bradshaw, P. 1985 The effect of convex surface curvature on turbulent boundary layers. J. Fluid Mech. 161, 347-369.

Mukund, R., Viswanath, P. R., Narasimha, R., Prabhu, A. \& Crouch, J. D. 2006 Relaminarization in highly favourable pressure gradients on a convex surface. J. Fluid Mech. 566, 97-115.

Nagarajan, S., Lele, S. K. \& Ferziger, J. H. 2007 Leading-edge effects in bypass transition. J. Fluid Mech. 572, 471-504.

Nicoud, Franck \& Ducros, FrÉdÉric 1999 Subgrid-scale stress modelling based on the square of the velocity gradient tensor. Flow Turbul. Combust. 62 (3), 183-200.

Nix, A. C. 2004 Effects of high intensity, large-scale freestream combustor turbulence on heat transfer in transonic turbine blades. PhD thesis, Virginia Polytechnic Institute and State University, Blacksburg, Virginia, US.

Ovchinnikov, V., Choudhari, M. M. \& Piomelli, U. 2008 Numerical simulations of boundary-layer bypass transition due to high-amplitude free-stream turbulence. J. Fluid Mech. 613, 135-169.

PAtel, V. C. 1965 Calibration of the preston tube and limitations on its use in pressure gradients. J. Fluid Mech. 23 (1), 185-208.

Pichler, R., Sandberg, R. D., Laskowski, G. \& Michelassi, V. 2017 High-fidelity simulations of a linear HPT vane cascade subject to varying inlet turbulence. In ASME Turbo Expo 201\%: Turbomachinery Technical Conference and Exposition, p. V02AT40A001. ASME.

SAndBerg, R. D. \& Michelassi, V. 2019 The current state of high-fidelity simulations for main gas path turbomachinery components and their industrial impact. Flow Turbul. Combust. 102 (4), 797-848.

Sandberg, R. D., Michelassi, V., Pichler, R., Chen, L. \& Johnstone, R. 2015 Compressible direct numerical simulation of low-pressure turbines-Part I: Methodology. J. Turbomach. 137 (5), 051011. 
SAndBerg, R. D. \& SAndham, N. D. 2006 Nonreflecting zonal characteristic boundary condition for direct numerical simulation of aerodynamic sound. AIAA J. 44 (2), 402-405.

Schlatter, P., Brandt, L., De Lange, H. C. \& Henningson, D. S. 2008 On streak breakdown in bypass transition. Phys. Fluids 20 (10), 101505.

Skote, M., Haritonidis, J. H. \& Henningson, D. S. 2002 Varicose instabilities in turbulent boundary layers. Phys. Fluids 14 (7), 2309-2323.

Smith, C. R. \& Metzler, S. P. 1983 The characteristics of low-speed streaks in the near-wall region of a turbulent boundary layer. J. Fluid Mech. 129, 27-54.

Spalart, P. R. \& Strelets, M. KH. 2000 Mechanisms of transition and heat transfer in a separation bubble. J. Fluid Mech. 403, 329-349.

Westin, K. J. A., Boiko, A. V., Klingmann, B. G. B., Kozlov, V. V. \& Alfredsson, P. H. 1994 Experiments in a boundary layer subjected to free stream turbulence. Part 1. Boundary layer structure and receptivity. J. Fluid Mech. 281, 193-218.

Wheeler, A. P. S., Sandberg, R. D.and Sandham, N. D., Pichler, R. \& Michelassi, V. 2016 Direct numerical simulations of a high-pressure turbine vane. J. Turbomach. 138 (7), 071003.

White, F. M. 1991 Viscous fluid flow. McGraw-Hill, New York.

Wu, X. \& Durbin, P. A. 2001 Evidence of longitudinal vortices evolved from distorted wakes in a turbine passage. J. Fluid Mech. 446, 199-228.

Wundrow, D. W. \& Goldstein, M. E. 2001 Effect on a laminar boundary layer of smallamplitude streamwise vorticity in the upstream flow. J. Fluid Mech. 426, 229-262.

ZAKI, T. A. 2013 From streaks to spots and on to turbulence: exploring the dynamics of boundary layer transition. Flow Turbul. Combust. 91 (3), 451-473.

Zaki, T. A., Wissink, J. G., Rodi, W. \& Durbin, P. A. 2010 Direct numerical simulations of transition in a compressor cascade: the influence of free-stream turbulence. J. Fluid Mech. 665, 57-98.

Zhao, Y., Xiong, S., Yang, Y. \& Chen, S. 2018 Sinuous distortion of vortex surfaces in the lateral growth of turbulent spots. Phys. Rev. Fluids 7 (3), 1-16.

ZhaO, Y., YANG, Y. \& CHEN, S. $2016 a$ Evolution of material surfaces in the temporal transition in channel flow. J. Fluid Mech. 793, 840-876.

ZhaO, Y., YANG, Y. \& ChEN, S. $2016 b$ Vortex reconnection in the late transition in channel flow. J. Fluid Mech. 802, R4. 


\section{University Library}

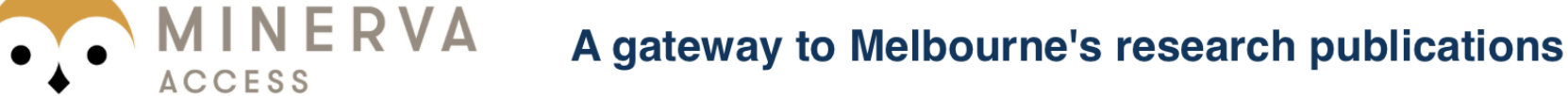

Minerva Access is the Institutional Repository of The University of Melbourne

Author/s:

Zhao, Y;Sandberg, RD

Title:

Bypass transition in boundary layers subject to strong pressure gradient and curvature effects

Date:

2020-04-10

\section{Citation:}

Zhao, Y. \& Sandberg, R. D. (2020). Bypass transition in boundary layers subject to strong pressure gradient and curvature effects. Journal of Fluid Mechanics, 888, https:// doi.org/10.1017/jfm.2020.39.

Persistent Link:

http://hdl.handle.net/11343/241857 